\title{
A model of the plasma flow and current in Saturn's polar ionosphere under conditions of strong Dungey cycle driving
}

\author{
C. M. Jackman and S. W. H. Cowley \\ Department of Physics and Astronomy, University of Leicester, Leicester LE1 7RH, UK \\ Received: (9 September 2005 - Revised: 16 January 2006 - Accepted: 16 March 2006 - Published: 19 May 2006
}

\begin{abstract}
We propose a simple model of the flow and currents in Saturn's polar ionosphere. This model is motivated by theoretical reasoning, and guided quantitatively by in situ field and flow data from space missions, ground-based IR Doppler measurements, and Hubble Space Telescope images. The flow pattern consists of components which represent (1) plasma sub-corotation in the middle magnetosphere region resulting from plasma pick-up and radial transport from internal sources; (2) the Vasyliunas-cycle of internal plasma mass-loss down the magnetospheric tail at higher latitudes; and (3) the polar Dungey-cycle flow driven by the solar wind interaction. Upstream measurements of the interplanetary magnetic field (IMF) indicate the occurrence of both extended low-field rarefaction intervals with essentially negligible Dungey-cycle flow, and few-day high-field compression regions in which the Dungey-cycle voltage peaks at a few hundred $\mathrm{kV}$. Here we model the latter conditions when the Dungey-cycle is active, advancing on previous axisymmetric models which may be more directly applicable to quiet conditions. For theoretical convenience the overall flow pattern is constructed by adding together two components - a purely rotational flow similar to previous axisymmetric models, and a sun-aligned twin vortex representing the dawn-dusk asymmetry effects associated with the Vasyliunas-and Dungey-cycle flows. We calculate the horizontal ionospheric current associated with the flow and the field-aligned current from its divergence. These calculations show that a sheet of upward-directed field-aligned current flows at the boundary of open field lines which is strongly modulated in local-time by the Dungey-cycle flows. We then consider implications of the field-aligned current for magnetospheric electron acceleration and aurorae using two plasma source populations (hot outer magnetospheric electrons and cool dense magnetosheath electrons). Both sources display
\end{abstract}

Correspondence to: C. M. Jackman

(cj47@ion.le.ac.uk) a strong dawn-dusk asymmetry in the accelerating voltages required and the energy fluxes produced, resulting from the corresponding asymmetry in the current. The auroral intensities for the outer magnetosphere source are typically $\sim 50 \mathrm{kR}$ at dawn and $\sim 5 \mathrm{kR}$ at dusk, in conformity with recent auroral observations under appropriate conditions. However, those for the magnetosheath source are much smaller. When the calculated precipitating electron energy flux values are integrated across the current layer and around the open closed field line boundary, this yields total UV output powers of $\sim 10 \mathrm{GW}$ for the hot outer magnetosphere source, which also agrees with observations.

Keywords. Magnetospheric physics (Current systems; Energetic particles, precipitating; Magnetospheric configuration and dynamics)

\section{Introduction}

Recent theoretical interest in the dynamics of Saturn's magnetosphere has been stimulated by new data from the Cassini Saturn orbiter space mission (e.g. Dougherty et al., 2005; Krimigis et al., 2005; Young et al., 2005), by ground-based IR Doppler measurements of ionospheric flows (Stallard et al., 2004), and by studies of the planet's aurorae using the Hubble Space Telescope (HST) (e.g. Gérard et al., 1995, 2004; Cowley et al., 2004a; Prangé et al., 2004). Coordinated HST and Cassini interplanetary observations have shown in particular that the aurorae respond strongly to upstream conditions, particularly the dynamic pressure of the solar wind (Clarke et al., 2005; Crary et al., 2005; Grodent et al., 2005; Bunce et al., 2005a), thus reflecting related behaviour observed in Saturn kilometric radiation (SKR) emissions (e.g. Desch, 1982; Desch and Rucker, 1983; Kurth et al., 2005; Jackman et al., 2005). For example, during extended solar wind rarefaction intervals in which the

Published by Copernicus GmbH on behalf of the European Geosciences Union. 
interplanetary magnetic field (IMF) is very weak, the aurorae are also typically weak, and consist of irregular patches which sub-corotate around the polar cap boundary. Following the arrival of corotating interaction region (CIR) compressions, however, the aurora are observed to brighten, to contract poleward, and to exhibit strong local-time asymmetry, with brightest aurorae occurring in the dawn sector. Cowley et al. (2005a) have suggested that the latter phenomena are associated with reconnection-related dynamics in Saturn's magnetosphere, in which intervals of rapid open flux closure in the tail are stimulated by sudden solar wind compressions, as is also sometimes observed to occur at Earth (e.g. Boudouridis et al., 2004, 2005). Corresponding dynamic behaviour has also been observed by Cassini directly in Saturn's nightside outer magnetosphere (Jackman et al., 2005; Bunce et al., 2005b). In the few-day strong-IMF compression regions that follow, strong nearcontinuous reconnection-related solar wind driving of the magnetosphere can then occur, in which net open flux is slowly accumulated in the tail (Badman et al., 2005). The corresponding aurorae are observed to remain bright, and to retain a strong dawn-dusk asymmetry. Weaker aurorae of a similar nature can also be observed during interplanetary rarefaction conditions of intermediate field strength.

These studies thus indicate that the solar wind interaction is important at Saturn, as it is at Earth (see, e.g. the review by Cowley et al., 2003). At the same time, it is also believed that internal dynamics are also significant, driven by the rapid $\sim 11 \mathrm{~h}$ planetary rotation and the internal sources of plasma formed by Saturn's and Titan's atmosphere and the surfaces of icy moons and ring material (Richardson et al., 1998; Saur et al., 2004). Recently, a qualitative picture of the overall plasma flow and electric current system in Saturn's coupled solar wind-magnetosphere-ionosphere system has been discussed by Cowley et al. (2004a), which contains co-existing elements related both to internally-driven processes and the solar wind interaction. This picture forms the basis of the theoretical model of the flow and current in Saturn's polar ionosphere developed here. A related quantitative model based on this picture was presented previously by Cowley et al. (2004b). However, for simplicity of initial discussion, this model assumed axi-symmetry of the system, such that only the basic latitudinal variations of the azimuthal flow were represented. A related axi-symmetric model of Jupiter's ionospheric flow and currents has also been proposed (Cowley et al., 2005b). When internally-driven and solar wind-related processes are both active in the magnetosphere, however, significant dawn-dusk asymmetries will also generally be present, as discussed qualitatively by Cowley et al. (2004a). The purpose of the present paper, therefore, is to present a more realistic model of Saturn's polar ionospheric flows and currents when both solar wind and internally-driven processes are active, thus generalising the initial axi-symmetric model of Cowley et al. (2004b). Specifically, we choose to model conditions of strong steady solar wind driving, that are most applicable to few-day strong-field interplanetary compression regions. We begin in the next section by outlining the overall physical picture presented by Cowley et al. (2004a), which forms the background of the detailed theoretical model which follows in Sects. 3 and 4.

\section{Physical picture}

As indicated above, the theoretical model developed here is based on the qualitative picture discussed by Cowley et al. (2004a), which contains co-existing features associated with both internally-driven processes and the solar wind interaction. Here we begin by outlining the overall physical picture, which provides motivation for the theory that follows in Sects. 3 and 4. This is illustrated in Fig. 1, where we show the flow in the magnetospheric equatorial plane (Fig. 1a) and in the northern polar ionosphere (Fig. 1b). In both figures the direction toward the Sun is at the bottom of the diagram, dusk is to the right, and dawn to the left.

Examining first the equatorial flows (Fig. 1a), the outer boundary of the diagram is the planetary magnetopause, extending to $\sim 20$ planetary radii $\left(R_{S}\right.$, conventionally equal to $60330 \mathrm{~km}$ ) in the sunward direction, while the arrowed solid lines within the boundary show plasma streamlines. With increasing radial distance from the planet, the regimes of plasma flow are characterised as follows. In the inner part of the magnetosphere the plasma to a first approximation corotates with the planet, due to the torque imposed by ion-neutral collisions at the feet of the field lines in the ionosphere. Voyager observations show that the rotation is near-rigid out to equatorial radial distances of $\sim 5 R_{S}$, after which the plasma angular velocity falls to about half of rigid rotation within several $R_{S}$ of the dayside magnetopause (Richardson, 1986, 1995; Richardson and Sittler, 1990). The fall in angular velocity is believed to be due to the production and pick-up of plasma from internal sources as mentioned above (e.g. Richardson et al., 1998), together with subsequent diffusive radial transport and loss, as discussed for Jupiter by Hill (1979) and Pontius and Hill (1982), and for Saturn by Saur et al. (2004). Here we term the near-rigidly rotating inner region the "inner magnetosphere", and the subcorotating region the "middle magnetosphere" respectively.

The latter region is then surrounded by an "outer" layer, bounded by the arrowed dashed streamlines, in which dynamical mass-loss of internal plasma takes place. In this region, the mass-loaded closed field lines which are rotating towards midnight from dusk extend into the tail and then pinch off to form a tailward-moving plasmoid, as first described by Vasyliunas (1983) in the jovian context. The "X"-line of the plasmoid is shown by the dashed line marked by Xs in the dusk-sector tail, while the "O"-line at its centre (a streamline) is indicated by the arrowed dashed line marked "O". The outer boundary of the plasmoid is indicated by the dotdashed line marked "P" (not a streamline), which eventually 

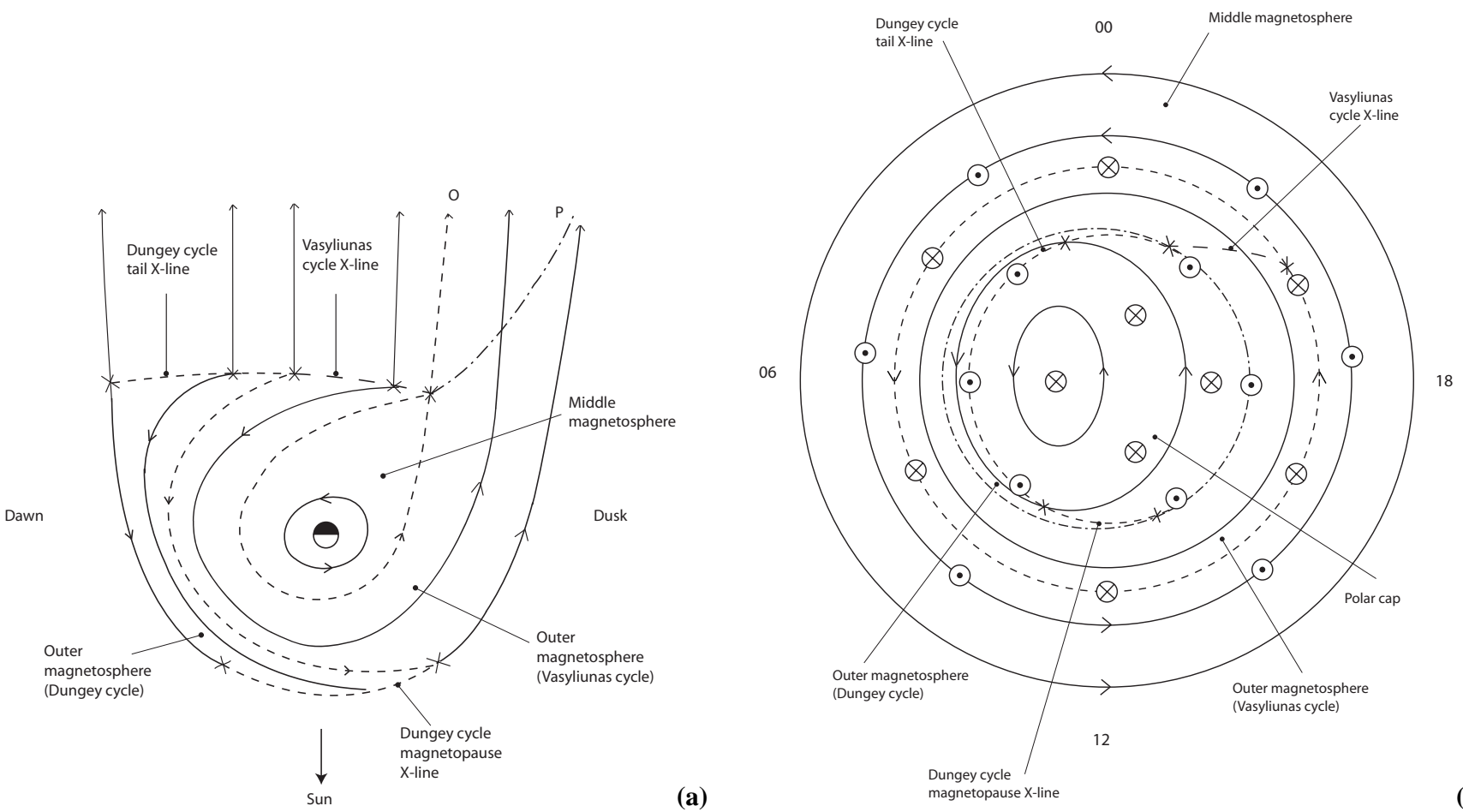

Fig. 1. (a) Sketch of the plasma flow in the equatorial plane of Saturn's magnetosphere where both steady-state Vasyliunas-cycle flow and Dungey-cycle flow are active. The direction to the Sun is at the bottom of the figure, dusk to the right, and dawn to the left. The outer boundary of the diagram represents the planet's magnetopause. Arrowed solid lines show plasma streamlines, while the inner and outer arrowed short-dashed lines (also streamlines) show the boundaries between the sub-corotating middle magnetosphere and the outer magnetosphere containing the Vasyliunas-cycle flow, and the latter and the Dungey-cycle flow, respectively. The heavy dashed line with Xs on the nightside shows the tail reconnection line associated with the Vasyliunas cycle, while in the tailward region the dashed line marked "O" shows the path of the plasmoid O-line formed by the reconnection (also a streamline), while "P" marks the outer limit of the plasmoid field lines (not a streamline) which eventually asymptotes to the dusk tail magnetopause. The nightside dashed line with Xs indicates the tail reconnection region associated with the Dungey cycle, which is shown contiguous with the Vasyliunas-cycle X-line, though this is not necessarily the case. The closed flues tubes formed at this reconnection region flow sunward via dawn toward an active magnetopause reconnection region in the noon sector (dashed line with Xs in the dayside sector), where they become open and move poleward out of the plane of the diagram. (After Cowley et al.,2004a). (b) Sketch of the plasma flow in Saturn's northern ionosphere for the case in which steady-state Vasyliunas-cycle flow and Dungey-cycle flow are active, as in Fig. 1a. The direction to the Sun is again at the bottom of the figure, dusk to the right, and dawn to the left. The outermost circle corresponds to a co-latitude of $\sim 30^{\circ}$ from the pole, which maps to the equatorial plane in the inner magnetosphere at a radial distance of $\sim 3 R_{S}$, poleward of which lies the sub-corotating flow of the middle magnetosphere. The pair of dashed lines poleward of this show the ring of closed flux tubes corresponding to the outer magnetosphere, which contains the Vasyliunas-cycle flow at lower latitudes, and the Dungey-cycle "return" flow in the dawn sector, flowing between the "merging gaps" on the open field boundary (marked by the Xs on the poleward dashed line). On open field lines within the inner dashed line, the flow consists of a slow rotation driven by ion-neutral collisions, and an anti-sunward flow between the merging gaps associated with the Dungey cycle. Overall, the equatorward boundary of Dungey-cycle flow is indicated by the dot-dashed line, marking its interface with the Vasyliunas-cycle flow. The circled dots and crosses indicate regions of upward and downward field-aligned current, respectively, as indicated by the divergence of the horizontal ionospheric current. Hall currents flow generally anti-clockwise round the pole and close in the ionosphere, while Pedersen currents flow generally equatorward and close in the field-aligned current systems shown. (After Cowley et al., 2004a)

asymptotes to the dusk magnetopause. After reconnection, the mass-reduced closed flux tubes contract back toward the planet at higher speeds, and rotate around to the dayside via dawn under the action of the ionospheric torque. As they do so, they become slowed and re-loaded with planetary plasma due to cross-field diffusion from the middle-magnetosphere, such that the process then repeats as the flux tubes rotate toward the tail via dusk. This flow is termed the "Vasyliunas cycle", and is conceived here for simplicity as a steady-state process. It also seems possible, however, that the process 
may be episodic, involving intervals of plasma accumulation in the sub-corotating outer magnetosphere region, followed by intervals of plasmoid-formation and mass-loss down the dusk tail flank.

The outermost region on the dawn side in Fig. 1a then contains the flows associated with the solar wind interaction, as first described by Dungey (1961) in the terrestrial context. Reconnection at the magnetopause $\mathrm{X}$-line, shown by the dashed line with Xs on the dayside boundary, produces open field lines which move poleward out of the plane of the diagram, and are stretched by the solar wind flow into a long nightside magnetic tail. This process occurs principally when the interplanetary magnetic field (IMF) points north, opposite to the southward-directed near-equatorial planetary field. The open tubes then reconnect again in the centre plane of the tail, principally on the dawn side as shown in the diagram, owing to the presence of the Vasyliunas-cycle outflow at dusk. The closed field lines produced by this process then flow back to the dayside again via dawn, acted on by the planetary ionospheric torque, where the process repeats. We term this flow the "Dungey cycle". The flow speed in the dawn-side layer is likely to be comparable to that in the adjacent Vasyliunas cycle layer, since neither region of closed flux will be strongly mass-loaded with planetary plasma. As a consequence we here consider the Dungeycycle "return" flow and the Vasyliunas-cycle flow to form a combined "outer magnetosphere" region, located between the sub-corotating flow of the middle magnetosphere and the magnetopause.

In Fig. 1b we show the corresponding flow in Saturn's northern polar ionosphere. The outer boundary of this diagram corresponds to a co-latitude from the northern pole of $\sim 30^{\circ}$, mapping magnetically into the near-rigidly corotating equatorial flow of the inner magnetosphere at a radial distance of $\sim 3 R_{S}$. The plasma angular velocity then falls with increasing latitude in the region mapping to the middle magnetosphere, reaching about half of rigid corotation at the boundary of the outer magnetosphere containing the Vasyliunas cycle flow, indicated by the outer arrowed dashed streamline. The outer magnetosphere region then lies between the dashed line rings, where the poleward dashed line corresponds to the open-closed field line boundary. This region contains the Vasyliunas-cycle flow in the lower-latitude portion, and the Dungey-cycle "return" flow at higher latitudes in the dawn sector, the latter flowing from the nightside to the dayside reconnection regions (or "merging gaps") lying on the open-closed field line boundary (dashed lines with Xs). In the dawn sector, the flows in this region are expected to be higher than in the adjacent middle magnetosphere due to the lower mass-loading of these flux tubes, as indicated above, but will slow to a comparable speed to the middle magnetosphere in the dusk sector due to subsequent diffusive mass loading in the Vasyliunas cycle.

The central region of open flux in Fig. 1b, which could not be represented in the equatorial diagram in Fig. 1a, extends to a typical co-latitude of $\sim 15^{\circ}$ according to Voyager magnetic field estimates (Ness et al., 1981; Cowley et al., 2004b). In the absence of on-going reconnection, the flow in this region is expected to consist of a rotational circulation driven by ion-neutral collisions in the ionosphere which twists the open tail field lines (Isbell et al., 1984). The plasma angular velocity to which this process gives rise depends on the poorly-known value of the effective ionospheric Pedersen conductivity, as will be discussed further below. Recent ground-based Doppler observations of ionospheric IR emissions by Stallard et al. (2004) indicate plasma angular velocities of about a third of rigid corotation in this region, this value implying an effective Pedersen conductance of the polar ionosphere of $\sim 0.5-1$ mho. Values of $\sim 1-2$ mho have also been derived for the middle magnetosphere region by Bunce et al. (2003), based on Voyager plasma angular velocity profiles and the observed sweep-back of middle magnetosphere magnetic field lines. When Dungey-cycle flow is also present, anti-sunward flow also occurs between the "merging gaps" on the open-closed field line boundary, adding vectorially to the sub-corotational flow just described, as shown in Fig. 1b. Some closed streamlines may continue to be present in the polar cap as shown, however, where open flux circulates between more major intervals of tail reconnection. Overall, the region containing the Dungey-cycle flow is that lying poleward of the dot-dashed line. This line coincides with the open-closed field line boundary in the dusk sector, and separates the Dungey-cycle "return" flow from the Vasyliunas-cycle flow on closed field lines in the dawn sector outer magnetosphere region.

We now consider the flux transport in these processes. Jackman et al. (2004) have recently estimated the flux transport in the Dungey cycle at Saturn. They used Cassini measurements of the upstream IMF, and an algorithm validated at Earth (e.g. Milan et al., 2004) and modified to kronian conditions. While the average Dungey-cycle voltage was estimated to be $\sim 50 \mathrm{kV}$ (corresponding to a flux transport of $\sim 50 \mathrm{kWb} \mathrm{s}^{-1}$ by Faraday's law), the results indicate a very variable interaction overall, modulated by the CIR structures in the solar wind outlined in the introduction. Conditions were found to vary between extended intervals of essentially negligible flux transport in low-IMF solar wind rarefaction regions, to intervals in which the Dungey-cycle voltage peaked at a few hundred $\mathrm{kV}$ in high-IMF compression regions. Here we choose to model compression-region conditions when the Dungey cycle is the most active, and have thus employed a Dungey-cycle voltage of $200 \mathrm{kV}$ in the illustrative model to be presented below (see also Badman et al., 2005). The flux transport in the Vasyliunas cycle at Saturn is not known at present, but for definiteness we assume it here to be comparable in magnitude to that of the active Dungey cycle, as will be discussed further below.

We now briefly consider the ionospheric and field-aligned current systems that are implied by these flows, and the relationship of the latter to Saturn's aurorae. The generally 
sub-corotating plasma flow in the ionosphere drives currents which are proportional to the degree of sub-corotation (i.e. to the electric field in the rest frame of the neutral atmosphere), the Pedersen currents flowing equatorward and the Hall currents eastward. While the Hall currents are thus expected to close in the ionosphere as a first approximation, the Pedersen currents instead close in the magnetosphere via rings of field-aligned current which are determined by the plasma angular velocity profile. These are indicated by circled dots and crosses in Fig. 1b, where the circled dots indicate upward current, and the circled crosses downward current. Four main regions of current are implied by the above discussion. At highest latitudes, distributed downward-directed fieldaligned current is expected within the polar cap, associated with the twisting of the tail lobe field lines mentioned above, that feeds the equatorward-directed ionospheric Pedersen current which grows with distance from the rotation axis. Assuming an effective ionospheric conductance of $\sim 1 \mathrm{mho}$, the field-aligned current densities were found to be $\sim 15 \mathrm{nA} \mathrm{m}^{-2}$ in the model developed by Cowley et al. (2004b). Much of this current then returns up the field lines in a narrow layer at the open-closed field line boundary, where the plasma angular velocities suddenly increase from about a third of rigid corotation on open field lines to an angular velocity significantly closer to rigid corotation on closed field lines in the outer magnetosphere, thus suddenly reducing the equatorward ionospheric Pedersen current. Assuming a current layer of a few hundred $\mathrm{km}$ thickness in the ionosphere as observed (i.e. some fraction of a degree of latitude), the peak upward current densities are estimated to be $\sim 50-200 \mathrm{nA} \mathrm{m}^{-2}$ by Cowley et al. (2004a, b), sufficient to require downward acceleration of magnetospheric electrons through field-aligned voltages of a few tens of $\mathrm{kV}$, resulting in the formation of $\mathrm{UV}$ aurorae of several tens of $\mathrm{kR}$ intensity. On this basis Cowley et al. (2004a,b) proposed that the "main oval" aurorae at Saturn (e.g. Gérard et al., 1995, 2004; Trauger et al., 1998) are associated with the boundary between open and closed field lines. In the situation depicted in Fig. 1b, however, the flow shear across the boundary is greater at dawn than at dusk, such that the upward current at the boundary and the aurorae will also be stronger at dawn than at dusk. It is the representation and quantification of these dawn-dusk effects that is the principal focus of the present paper. As mentioned in the introduction, dawn-dusk asymmetries are a commonly observed but not ubiquitous feature of Saturn's aurorae.

At lower latitudes within the outer magnetosphere region the field-aligned current then reverses once more to downward, particularly at its equatorward boundary at dawn where the angular velocity is expected to drop with increasing colatitude into the middle magnetosphere, thus implying an increase in the equatorward Pedersen current. These downward currents will be less significant at dusk, however, where the shear in the flow between the outer and middle magnetosphere regions is expected to be less. Finally, at lower latitudes in the middle magnetosphere, the field-aligned current once more becomes directed upward as the plasma angular velocities increase toward rigid corotation and the Pedersen current falls with increasing co-latitude. This middle magnetosphere current system was modelled by Cowley and Bunce (2003) and Cowley et al. (2004b) based on Voyager plasma angular velocity profiles, showing that for an ionospheric Pedersen conductance of $\sim 1$ mho, the large-scale upward current density peaks at $\sim 10-15 \mathrm{nA} \mathrm{m}^{-2}$ at $\sim 20^{\circ}$ colatitude. Such current densities are too low to require downward acceleration of hot magnetospheric electrons into the ionosphere, such that these currents are not expected to be associated with strong auroral emissions. Smaller-scale flow features are also observed in some of the Voyager data which may be associated with larger amplitude but more localised current systems. However, modelling of these features by Cowley and Bunce (2003) still led only to weak precipitation and auroras in this lower-latitude region. These considerations do not, therefore, alter our previous conclusion that Saturn's aurorae are associated with the open-closed field line boundary, and hence with the solar wind interaction, rather than the middle magnetosphere and internal dynamics. At Jupiter, however, the equivalent middle magnetosphere "corotation breakdown" currents are more intense by at least an order of magnitude (e.g. Cowley et al., 2005b), and are believed to be associated with accelerated electron precipitation that produces the bright "main oval" auroral emissions in that case (Bunce and Cowley, 2001; Cowley and Bunce, 2001; Hill, 2001; Southwood and Kivelson, 2001; Grodent et al., 2003).

\section{Basic theory}

In this section we present the basic theory which underlies our model of plasma flow and currents in Saturn's ionosphere. Three basic ingredients are required. The first is the choice of the geometry of the ionospheric layer where the horizontal currents flow, and of the planetary magnetic field that threads through it. The second is the flow of plasma and neutral atmosphere in this layer, from which the horizontal and field-aligned currents can be calculated. The third is the model basis on which auroral parameters are calculated from the field-aligned currents. Each of these topics will now be discussed in turn. The specifics of the model will then be given in Sect. 4.

\subsection{Geometry and magnetic field model}

With regard to the first of these topics, the models presented by Cowley and Bunce (2003) and Cowley et al. (2004b) employed a spheroidal ionospheric layer following the flattened figure of the planet, the full SPV model of the planetary magnetic field which is axially symmetric about the planet's spin axis (Davis and Smith, 1990), and a modest correction for the consequent fact that the magnetic field does not then pass 
exactly normally through the ionospheric current layer. Here, however, we relax these features in order to incorporate more complex local-time dependent flows into the model while retaining simplicity of calculation. Specifically, we first take the polar ionospheric layer to be at a fixed radial distance of $R_{i}=55364 \mathrm{~km}$, corresponding to an altitude of $1000 \mathrm{~km}$ above the 1 bar reference spheroid of the planet at the pole (e.g. Trauger er al., 1998). This value compares with a corresponding equatorial ionospheric radius of $61,268 \mathrm{~km}$, and the conventional ( 1 bar) Saturn radius $R_{S}=60330 \mathrm{~km}$ mentioned in the introduction. Using the full spheroidal geometry, the increase in ionospheric radius between the pole and $20^{\circ}$ co-latitude, bounding the main region of interest here, is only $\sim 1 \%$, so this does not represent a major simplification. Second, we also assume that the planetary magnetic field is purely radial through this spherical surface, and thus neglect the correction for non-vertical magnetic fields. We note that the tilt of the SPV field to the ionospheric normal varies from zero at the pole to a maximum of $\sim 18^{\circ}$ at $20^{\circ}$ co-latitude. However, the latter tilt changes the horizontal current by only $\sim 5 \%$, so that this is also a modest effect. Third, although we could here take any axi-symmetric radial field varying with co-latitude without changing the principle of the calculations, for full simplicity we have taken the polar field to be of constant strength $B_{i}$ over the whole region of interest. In the SPV model, the radial field in the northern hemisphere decreases from $\sim 75600 \mathrm{nT}$ at the pole to $\sim 66400 \mathrm{nT}$ at $20^{\circ}$ co-latitude, while in the Southern Hemisphere it varies from $\sim 62400$ to $\sim 55500 \mathrm{nT}$ over the same range. Here, therefore, for general calculations we have taken the polar radial field strength to be $B_{i}=64000 \mathrm{nT}$ as a round value representative of both polar hemispheres. Inter-hemispheric differences can still be investigated in principle, however, by using differing field strengths for the two polar regions.

It is also useful to introduce the flux function of the field, $F$, which for an axi-symmetric field is related to the field components (in spherical polar co-ordinates) by $\boldsymbol{B}=(1 / r \sin \theta) \nabla F \times \hat{\varphi}$. The properties of the flux function are such that $F=$ const defines a flux shell mapping from the northern to the Southern Hemisphere via the equator, thus allowing easy field line mapping between these regions, and that the amount of magnetic flux contained between the shells of flux functions $F_{1}$ and $F_{2}$, integrated in azimuth around the axis, is $2 \pi\left(F_{2}-F_{1}\right)$. For a spherical ionosphere and a constant radial field, the above expression relating the flux function to the field is readily integrated to give the ionospheric flux function as

$F\left(\theta_{i}\right)=B_{i} R_{i}^{2}\left(1-\cos \theta_{i}\right)$,

where we have taken $F$ to be zero on the magnetic axis.

\subsection{Plasma flow and current}

We now briefly give the basic equations which govern the plasma flow and current in our model calculations. The convective plasma flow, which applies to electrons throughout the ionosphere and also to ions above the layer where ion-neutral collisions occur (and where the ionospheric currents consequently flow), may be specified by defining either the ionospheric plasma velocity in the inertial frame, $\boldsymbol{V}_{i}$, or equivalently the corresponding electric field $\boldsymbol{E}_{i}$. These are related by

$\boldsymbol{V}_{i}=\frac{\boldsymbol{E}_{i} \times \boldsymbol{B}_{i}}{B_{i}^{2}}=\frac{\boldsymbol{E}_{i} \times \hat{\boldsymbol{r}}}{B_{i}}$

or

$\boldsymbol{E}_{i}=-\boldsymbol{V}_{i} \times \boldsymbol{B}_{i}=B_{i} \hat{\boldsymbol{r}} \times \boldsymbol{V}_{i}$,

where the second expressions are valid specifically in the northern ionosphere where $\boldsymbol{B}_{i}$ points radially outward. Since the ionospheric magnetic field is essentially incompressible, the curl of $\boldsymbol{E}_{i}$ must be zero by Faraday's law, so the electric field can also be expressed as the gradient of a scalar potential $\Phi_{i}$

$\boldsymbol{E}_{i}=-\nabla \Phi_{i}$.

The motion of the ionospheric plasma can thus be determined by specifying either $\boldsymbol{V}_{i}, \boldsymbol{E}_{i}$, or $\Phi_{i}$. Substitution of Eq. (3) into Eq. (2a) shows that the streamlines of the ionospheric flow are the equipotentials $\Phi_{i}=$ const, where the magnetic flux per unit time transported between streamlines of potentials $\Phi_{1}$ and $\Phi_{2}$ is $\left(\Phi_{2}-\Phi_{1}\right) \mathrm{Wb} \mathrm{s}^{-1}$.

While the above quantities are defined in the inertial frame, the horizontal ionospheric Pedersen and Hall currents are determined by the electric field in the rest frame of the neutral atmosphere in the ionospheric current layer, $\boldsymbol{E}_{i}^{\prime}$. Specifically, the height-integrated current intensities are given by

$\boldsymbol{i}_{P}=\Sigma_{P} \boldsymbol{E}_{i}^{\prime} \quad$ and $\quad \boldsymbol{i}_{H}=\Sigma_{H} \hat{\boldsymbol{r}} \times \boldsymbol{E}_{i}^{\prime}$,

where $\Sigma_{P}$ and $\Sigma_{H}$ are the Pedersen and Hall conductances (the conductivities integrated in height through the ionosphere) respectively. This electric field is related to the value in the inertial frame $\boldsymbol{E}_{i}$ by

$\boldsymbol{E}_{i}^{\prime}=-\left(\boldsymbol{V}_{i}-\boldsymbol{V}_{n}\right) \times \boldsymbol{B}_{i}=\boldsymbol{E}_{i}+\boldsymbol{V}_{n} \times \boldsymbol{B}_{i}$,

where $\boldsymbol{V}_{n}$ is the velocity of the neutral atmosphere in the inertial frame. We further assume for simplicity that the neutral atmosphere rigidly corotates with the planet at speed $\boldsymbol{V}_{R C}=R_{i} \Omega_{S} \sin \theta_{i} \hat{\varphi}$ in the inertial frame (where $\Omega_{S}$ is Saturn's angular velocity equal to $1.638 \times 10^{-4} \mathrm{rad} \mathrm{s}^{-1}$ ), except for a wind driven by ion drag which is a constant factor $k$ times the ion velocity in the corotating frame (see e.g. Huang and Hill (1989) and Millward et al. (2005) for discussions in a jovian context). That is, we assume $\boldsymbol{V}_{n}=\boldsymbol{V}_{R C}+k\left(\boldsymbol{V}_{i}-\boldsymbol{V}_{R C}\right)$, so that $\boldsymbol{V}_{i}-\boldsymbol{V}_{n}=(1-k)\left(\boldsymbol{V}_{i}-\boldsymbol{V}_{R C}\right)$. Substitution into Eq. (5) then yields

$\boldsymbol{E}_{i}^{\prime}=(1-k)\left(\boldsymbol{E}_{i}+B_{i} R_{i} \Omega_{S} \sin \theta_{i} \hat{\theta}\right)$, 
so that from Eq. (4) the ionospheric currents become

$\boldsymbol{i}_{P}=\Sigma_{P}^{*}\left(\boldsymbol{E}_{i}+B_{i} R_{i} \Omega_{S} \sin \theta_{i} \hat{\theta}\right)$

and

$\boldsymbol{i}_{H}=\Sigma_{H}^{*}\left(\hat{\boldsymbol{r}} \times \boldsymbol{E}_{i}+B_{i} R_{i} \Omega_{S} \sin \theta_{i} \hat{\varphi}\right)$,

where $\Sigma_{P}^{*}=(1-k) \Sigma_{P}$ and $\Sigma_{H}^{*}=(1-k) \Sigma_{H}$ are the "effective" Pedersen and Hall conductivities, respectively, reduced from the true values by "slippage" of the neutral atmosphere from rigid corotation. The field-aligned current density is then determined from the horizontal current intensity by current continuity as $j_{\| i}=-\operatorname{div}\left(i_{P}+i_{H}\right)$. For simplicity we take $\Sigma_{P}^{*}$ and $\Sigma_{H}^{*}$ to be constants in our model over the whole polar ionosphere. In this case we find that $\operatorname{div} i_{H}=0$, such that the Hall current closes wholly in the ionosphere, and the field-aligned current is determined only by the divergence of the Pedersen current. From Eq. (7a) we have

$$
\begin{aligned}
j_{\| i}= & -\operatorname{div} \boldsymbol{i}_{P}=-\Sigma_{P}^{*} \operatorname{div}\left(\boldsymbol{E}_{i}+B_{i} R_{i} \Omega_{S} \sin \theta_{i} \hat{\theta}\right)= \\
& \Sigma_{P}^{*}\left(\nabla^{2} \Phi_{i}-2 B_{i} \Omega_{S} \cos \theta_{i}\right) .
\end{aligned}
$$

In accordance with the results discussed in Sect. 2 and the previous studies by Cowley and Bunce (2003) and Cowley et al. (2004b), here we will take $\Sigma_{P}^{*}$ to have a value of 1 mho.

\subsection{Auroral parameters}

Having determined the field-aligned currents, we are then interested to consider their consequences for auroral precipitation. Specifically we are interested in the regions of upward field-aligned current which are expected to be carried mainly by precipitating magnetospheric electrons, and whether these currents require field-aligned acceleration of the "source" populations. To determine this, we compare the model values of the field-aligned current density with the maximum that can be carried by magnetospheric electrons without acceleration. This is given by

$j_{\| i 0}=e N\left(\frac{W_{t h}}{2 \pi m_{e}}\right)^{1 / 2}$,

where the magnetospheric electron population has been assumed to be an isotropic Maxwellian of density $N$ and thermal energy $W_{t h}$ (equal to $k_{B} T$ where $T$ is temperature and $k_{B}$ is Boltzmann's constant), and $e$ and $m_{e}$ are the electron charge and mass respectively. This expression corresponds to the case of a full downward-going loss-cone, and an empty upward-going loss-cone. If the model current density is higher than that given by Eq. (9), then according to Knight's (1973) kinetic theory a field-aligned voltage must exist along the field lines which accelerates magnetospheric electrons into the ionosphere to produce the required current. The minimum value of this voltage is given by

$\Phi_{\|}=\frac{W_{t h}}{e}\left[\left(\frac{j_{\| i}}{j_{\| i 0}}\right)-1\right]$, this value being appropriate if the "top" of the voltage drop is located at a radial distance well above the minimum value given by

$$
\left(\frac{r_{\min }}{R_{i}}\right) \approx\left(\frac{j_{\| i}}{j_{\| i 0}}\right)^{1 / 3} .
$$

In Eq. (11) we have assumed as a sufficient approximation that the field strength drops as the inverse cube of the radial distance along the polar field lines, corresponding to the planetary dipole field. Equation (10) also assumes that the voltage drop is sufficiently compact along the field lines that no electrons mirror before they have experienced the full voltage drop. Following Lundin and Sandahl (1978), the enhanced precipitating energy flux of the electrons is then given by

$$
E_{f}=\frac{E_{f 0}}{2}\left[\left(\frac{j_{\| i}}{j_{\| i 0}}\right)^{2}+1\right],
$$

where $E_{f 0}$ is the unaccelerated electron energy flux corresponding to Eq. (9), given by

$E_{f 0}=2 N W_{t h}\left(\frac{W_{t h}}{2 \pi m_{e}}\right)^{1 / 2}$.

These expressions will be used below to estimate the fieldaligned voltages, energy fluxes, and consequent UV auroral luminosities associated with the upward-directed currents in the model.

\section{Model of the plasma flow and current}

\subsection{Form of the plasma velocity model}

The model of the ionospheric flow presented here represents a development of the axi-symmetric models of Cowley and Bunce (2003) and Cowley et al. (2004b), constructed to provide a quantitative representation of the asymmetric ionospheric flow pattern depicted in Fig. 1b. Here we begin by providing an overview of the basis on which the model has been developed. We first assume for simplicity that the Vasyliunas cycle and the Dungey cycle return flow combine to produce an outer magnetosphere region which forms a symmetrical ring of closed flux centred on the pole, though containing faster flows at dawn than at dusk, as in Fig. 1b. If so, the polar cap region of open flux is also circular, and centred on the pole. The resulting concentric ring geometry of the flow regions then greatly simplifies the mathematics of the model. In our analysis we will denote quantities associated with the polar cap region by subscript "PC", the outer magnetosphere ring by "OM", and the middle magnetosphere region at lower latitudes by "MM".

Briefly, for theoretical convenience, the flow model is taken to consist of the sum of two components. The first is a 
rotational flow (denoted by subscript "RO") which is essentially similar to the axisymmetric model presented previously by Cowley et al. (2004b). That is, with increasing latitude on closed field lines, the plasma angular velocity falls from near-rigid corotation in the inner magnetosphere to $\sim 55 \%$ of rigid corotation at the outer boundary of the middle magnetosphere, and then increases to $75 \%$ of rigid corotation in the outer magnetosphere ring, these variations being in accord with the large-scale behaviour of the Voyager plasma velocity measurements (e.g. Richardson, 1986, 1995; Richardson and Sittler, 1990). In view of the previous results of Cowley and Bunce (2003) mentioned in Sect. 2, here we do not include representation of the smaller-scale flow features observed in some of those measurements. On open field lines the angular velocity then falls to $30 \%$ of rigid corotation, in accordance with the observations of Stallard et al. (2004) and the theory of Isbell et al. (1984) (for an effective ionospheric conductance of $1 \mathrm{mho}$ ). Flow asymmetry is then introduced by vectorially adding a second component consisting of a sun-aligned twin-vortex (denoted by subscript "TV"), which provides anti-sunward flow across the polar cap at a rate determined by the Dungey-cycle voltage, and return sunward flow via dawn and dusk which is confined to the outer magnetosphere ring. As indicated in Sect. 2, this voltage is chosen to be equal to $200 \mathrm{kV}$ corresponding to interplanetary compression region conditions, based on estimates by Jackman et al. (2004) and Badman et al. (2005). For simplicity, the twinvortex is chosen to be symmetrical about the noon-midnight meridian. The sunward "return" flow is thus the same at dawn and dusk, but adds vectorially to the rotational flow in the outer magnetosphere region to produce a combined flow which, though directed consistently eastward as in Fig. 1b, is increased to close to rigid corotation at dawn, but reduced to near outer middle magnetosphere values $(\sim 55 \%$ of rigid corotation) at dusk. Specifically, we choose the width of the outer magnetosphere ring such that the averaged angular velocity of the twin vortex flow at dawn and dusk corresponds to $20 \%$ of rigid corotation. If the flow associated with the rotational component is then $75 \%$ of rigid corotation, as indicated above, the combined flow in the outer magnetosphere ring will average to $95 \%$ of rigid corotation at dawn and 55\% at dusk, in conformity with the above discussion. With regard to the middle magnetosphere at lower latitudes, we note that (as indicated above) the return flow of the twin-vortex is confined wholly to the outer magnetosphere ring, so that only the rotational flow component contributes in this region.

Having thus outlined the nature of the flow, we now consider the location of the boundaries between the three flow regions, specified by the choice of boundary flux function values, related to co-latitude through Eq. (1). The boundary of the polar cap (denoted by subscript "BPC") is first chosen to correspond to flux function $F_{B P C}=1530 \mathrm{nT} R_{S}^{2}$, essentially the same as employed previously by Cowley et al. (2004b). The amount of open flux contained within the polar cap is then $2 \pi F_{B P C} \approx 35 \mathrm{GWb}$, a value consistent with in situ magnetic field data obtained from the Voyager spacecraft (Ness et al., 1981; Cowley et al., 2004b), and with the results of Badman et al. (2005) derived from HST images. From Eq. (1) the co-latitude of the polar cap boundary is then $\theta_{i B P C} \approx 13.68^{\circ}$. As indicated above, the location of the boundary of the outer magnetosphere region (denoted by subscript "BOM"), where it interfaces with the middle magnetosphere, is then chosen so that the averaged angular velocity within this region associated with the twin-vortex flow is $20 \%$ of rigid corotation. Since the total amount of magnetic flux in the outer magnetosphere ring is $2 \pi\left(F_{B O M}-F_{B P C}\right)$, it is easy to show that the flux per unit time transported by an azimuthal plasma flow of angular velocity $\omega$ in the inertial frame is just $\omega\left(F_{B O M}-F_{B P C}\right)$, equal to the voltage associated with the electric field across the ring in that frame. Since the voltage associated with the "return" flow of the twin vortex at both dawn and dusk is just half the Dungey-cycle voltage $\Phi_{D C}$ (the voltage associated with the antisolar transport across the polar cap), we thus have

$$
\left(F_{B O M}-F_{B P C}\right)=\frac{\Phi_{D C}}{2\langle\omega\rangle_{T V}},
$$

where $\Phi_{D C}$ is chosen to be $200 \mathrm{kV}$ as indicated above, and the averaged angular velocity associated with the twin-vortex return flow is chosen to be $\langle\omega\rangle_{T V}=0.2 \Omega_{S}$. From Eq. (14) we then find $F_{B O M} \approx 2368.67 \mathrm{nT} R_{S}^{2}$, corresponding to a colatitude of $\theta_{\text {B }} O M=17.05^{\circ}$, such that the amount of closed flux in the outer magnetosphere ring is $\sim 19 \mathrm{GWb}$. We note that the flux function value of the corresponding feature in the axisymmetric Cowley et al. (2004b) model based solely on Voyager data is $2200 \mathrm{nTRs}^{2}$, similar to the value employed here.

We can now also derive the value of the voltage (flux transport) associated with the Vasyliunas cycle in the model. On a similar basis to the above, the voltage across the outer magnetosphere ring associated with the rotational flow component is $\triangle \Phi_{R O O M}=\omega_{O M}\left(F_{B O M}-F_{B P C}\right)$, where $\omega_{O M}=0.75 \Omega_{S}$ is the angular velocity associated with this flow component in this region, as above. Substituting from Eq. (14) we then find

$\Delta \Phi_{R O O M}=\frac{\omega_{O M}}{2\langle\omega\rangle_{T V}} \Phi_{D C}$,

so that with the above numbers we have $\Delta \Phi_{R O} O M=375 \mathrm{kV}$. The combined voltage at dusk, equal to the voltage in the Vasyliunas cycle (Fig. 1b), is then

$\Phi_{V C}=\Delta \Phi_{R O O M}-\frac{\Phi_{D C}}{2}=275 \mathrm{kV}$,

comparable with, but slightly larger than the Dungeycycle voltage. The combined voltage at dawn is similarly $\Delta \Phi_{R O O M}+\left(\Phi_{D C} / 2\right)=475 \mathrm{kV}$, equal to the sum of the Dungey-cycle and the Vasyliunas-cycle voltages (see Fig. 1b). 
The above discussion has thus provided an overview of how the flow model is constructed, and how its major parameters have been determined. Below we provide a systematic quantitative description of its components, and begin with the rotational flow.

\subsection{Rotational flow model}

The rotational flow is specified by defining the plasma angular velocity as a function of the flux function, $\omega_{R} O=\omega_{R} O(F)$. In conformity with the discussion above we choose for the polar cap

$\omega_{R O}(F)=\omega_{P C}=0.3 \Omega_{S}$,

while for the outer magnetosphere

$\omega_{R O}(F)=\omega_{O M}=0.75 \Omega_{S}$,

and for the middle magnetosphere

$\omega_{R O}(F)=$

$$
\Omega_{S}-\left(\Omega_{S}-\omega_{B M M}\right) \frac{\left(1+\left(F_{B O M} / F_{M M}\right)^{n}\right)}{\left(1+\left(F / F_{M M}\right)^{n}\right)} .
$$

The last expression is based on the overall plasma angular velocity profiles observed during the Voyager fly-bys, as employed previously by Cowley and Bunce (2003) and Cowley et al. (2004b), and is such that the angular velocity in the middle magnetosphere at the BOM boundary is $\omega_{R O}\left(F_{B O M}\right)=\omega_{B M M}$, and then increases to rigid corotation, $\omega_{R O}=\Omega_{S}$, as $F$ becomes large. The change from sub-corotation at angular frequency $\omega_{B M M}$ to rigid corotation takes place in the vicinity of $F \approx F_{M M}$, and the change is increasingly sharp as the exponent $n$ increases. The specific values employed here are $\omega_{B M M} \approx 0.587 \Omega_{S}$, $F_{M M}=3600 \mathrm{nT} R_{S}^{2}$, and $n=8$ (Cowley et al., 2004b). The value of $\omega_{B M M}$ has been chosen so that there is no discontinuity in the combined flow (rotational plus twin vortex) at the dusk "BOM" boundary between the outer and middle magnetosphere.

The associated ionospheric plasma velocity and electric field in the inertial frame (Eq. (2b)) are then

$V_{i R O}=R_{i} \sin \theta_{i} \omega_{R O} \hat{\varphi}$

and

$E_{i R O}=-B_{i} R_{i} \sin \theta_{i} \omega_{R O} \hat{\theta}$

while from Eq. (3) the electrostatic potential, assumed zero on the axis, is

$\Phi_{R O}(F)=\int_{0}^{F} \omega\left(F^{\prime}\right) d F^{\prime}$, a form which is generally valid for an axi-symmetric field and flow. Due to the constant values of plasma angular velocity in the polar cap and outer magnetosphere assumed above, the expressions for the electrostatic potential in these regions are especially simple. For the polar cap we have

$\Phi_{R O}(F)=\omega_{P C} F$,

while for the outer magnetosphere

$\Phi_{R O}(F)=\omega_{O M}\left(F-F_{B P C}\right)+\omega_{P C} F_{B P C}$.

With the above values, the voltage across the polar cap associated with the rotational flow in the inertial frame is thus $\sim 273.6 \mathrm{kV}$, comparable to the Dungey- and Vasyliunas-cycle values, while that across the outer magnetosphere is $375 \mathrm{kV}$ as previously indicated.

\subsection{Twin-vortex flow model}

The twin-vortex flow is described by specifying an electrostatic potential as a function of co-latitude $\theta_{i}$ and azimuthal angle $\varphi_{i}$ on the ionospheric sphere, $\Phi_{i T V}\left(\theta_{i}, \varphi_{i}\right)$, which, when added to the rotational potential given by Eq. (20), gives the total potential, and hence the electric field and flow of the model. Azimuth $\varphi_{i}$ increases with local time, and is defined such that $\varphi_{i}=0^{\circ}$ points towards the Sun. In common with previous derivations of related twin-vortex flows in the terrestrial system (see below), we look for potentials which are solutions of Laplace's equation on a spherical surface

$$
\begin{aligned}
& \nabla^{2} \Phi_{i T V}=\frac{1}{R_{i}^{2} \sin ^{2} \theta_{i}} \\
& \quad\left[\sin \theta_{i} \frac{\partial}{\partial \theta_{i}}\left(\sin \theta_{i} \frac{\partial \Phi_{i T V}}{\partial \theta_{i}}\right)+\frac{\partial^{2} \Phi_{i T V}}{\partial^{2} \varphi_{i}}\right]=0 .
\end{aligned}
$$

Physically acceptable solutions are obtained separately for the polar cap and the outer magnetosphere, where the outer boundary of the latter region is taken to be an equipotential (i.e. a streamline), such that the twin-vortex flow does not extend into the middle magnetosphere at lower latitudes as previously stated. The solutions in the polar cap and outer magnetosphere regions are then joined by specifying a common distribution of potential around the polar cap (BPC) boundary. We note from Eq. (8) that the effect of these assumptions is to confine the field-aligned currents associated with the twin-vortex flow to sheet currents lying on the BPC and BOM boundaries. Following the formulation of Freeman and Southwood (1988) and Freeman et al. (1991), to solve Eq. (21) we change variable from $\theta_{i}$ to $x_{i}$ given by

$x_{i}=\log _{e}\left(\tan \frac{\theta_{i}}{2}\right) \quad$ so that $\sin \theta_{i} \frac{\partial}{\partial \theta_{i}} \equiv \frac{\partial}{\partial x_{i}}$,

and look for separable solutions. The required solutions are as follows. For $x_{i} \leq x_{i}$ B PC in the polar cap we have

$\Phi_{i T V}\left(x_{i}, \varphi_{i}\right)=$
$\sum_{m=1}^{\infty}\left(a_{m} \sin m \varphi_{i}+b_{m} \cos m \varphi_{i}\right) \exp \left(m\left(x_{i}-x_{i}\right.\right.$ BPC $\left.)\right)$, 
while for $x_{i B P C} \leq x_{i} \leq x_{i \text { BOM }}$ in the outer magnetosphere

$$
\begin{aligned}
& \Phi_{i T V}\left(x_{i}, \varphi_{i}\right)= \\
& \sum_{m=1}^{\infty}\left(a_{m} \sin m \varphi_{i}+b_{m} \cos m \varphi_{i}\right) \frac{\sinh \left(m\left(x_{i B O M}-x_{i}\right)\right)}{\sinh \left(m\left(x_{\text {B BOM }}-x_{\text {B BPC }}\right)\right)} .
\end{aligned}
$$

In these expressions $x_{i B P C}$ and $x_{i B O M}$ are the values of $x_{i}$ on the BPC and BOM boundaries, respectively, defined by the flux function values of the boundaries, $F_{B P C}$ and $F_{B O M}$, and the corresponding co-latitudes of the boundaries, $\theta_{i} B P C$ and $\theta_{i \text { B } O M}$ obtained from Eq. (1). The expressions are such that on the BOM boundary we have $\Phi_{i T V}\left(x_{i} B O M, \varphi_{i}\right)=0$ for all $\varphi_{i}$, as required (i.e. the BOM boundary is an equipotential), while on the BPC boundary Eqs. (23) and (24) both give

$\Phi_{i T V}\left(x_{i B P C}, \varphi_{i}\right)=\sum_{m=1}^{\infty}\left(a_{m} \sin m \varphi_{i}+b_{m} \cos m \varphi_{i}\right)$,

which expresses the boundary potential as a Fourier series in $\varphi_{i}$. We can then choose any single-valued function for the distribution of potential around the boundary, $\Phi_{i T V}\left(x_{i B P C}, \varphi_{i}\right)$, and solve for the required coefficients $a_{m}$ and $b_{m}$ through the usual Fourier integrals

$a_{m}=\frac{1}{\pi} \int_{-\pi}^{\pi} d \varphi_{i} \Phi_{i T V}\left(x_{i B P C}, \varphi_{i}\right) \sin m \varphi_{i}$

and

$b_{m}=\frac{1}{\pi} \int_{-\pi}^{\pi} d \varphi_{i} \Phi_{i T V}\left(x_{i B P C}, \varphi_{i}\right) \cos m \varphi_{i}$.

(Note that the arbitrary zero of potential has been chosen so that the averaged value of $\Phi_{i T V}\left(x_{i B P C}, \varphi_{i}\right)$ around the boundary is zero. Any common constant potential value can be added to those given by Eqs. (23)-(25) if this is not the case.) Equations (23) and (24) then give the required solutions in the polar cap and outer magnetosphere for the given distribution of potential on the BPC (open-closed field line) boundary.

We thus now consider the potential on this boundary, and derive the corresponding Fourier coefficients. We first note from Eq. (26b) that if the twin-vortex flow is symmetric about the noon-midnight meridian, as will be assumed here, then the $b_{m}$ coefficients are zero for all $m$. Furthermore, if the pattern is also symmetric about the dawn-dusk meridian, as will also be assumed for simplicity, then the only non-zero $a_{m}$ are those with $m$ odd. The simplest choice would be to take $a_{1}=\Phi_{D C} / 2$, where $\Phi_{D C}$ is the total twin-vortex voltage across the polar cap driven by the Dungey cycle, with all other $a_{m}$ zero, giving a very broad distribution of flow into and out of the polar cap about noon and midnight respectively. More realistically, however, the flow into (and out of) the polar cap may be more restricted in local time about noon (and midnight), giving rise to a "merging gap" flow configuration. Here we therefore take for the "afternoon" quadrant $0 \leq \varphi_{i} \leq \pi / 2$

$\Phi_{i T V}\left(\theta_{i B P C}, \varphi_{i}\right)=$

$$
\frac{\Phi_{D C}}{2}\left\{\begin{array}{cc}
\sin \left(\frac{\pi \varphi_{i}}{\varphi_{M G}}\right) & \text { for } 0 \leq \varphi_{i} \leq \frac{\varphi_{M G}}{2} \\
1 & \text { for } \frac{\varphi_{M G}}{2} \leq \varphi_{i} \leq \frac{\pi}{2}
\end{array}\right\},
$$

with corresponding expressions for the other quadrants. With Eq. (27), flow takes place across the boundary only in azimuthal sectors of total angular width $\varphi_{M G}$ about noon (and midnight), and is zero (the boundary is "adiaroic") elsewhere. The functional form chosen avoids a discontinuity in the first derivative of the potential (i.e. in the azimuthal component of the electric field and hence the north-south flow) at the junction between the merging gap and the adiaroic section of the boundary. Introducing Eq. (27) into Eq. (26a) then yields for $m$ odd

$a_{m}=\frac{4}{\pi} \int_{0}^{\pi / 2} d \varphi_{i} \Phi_{i T V}\left(\theta_{i B P C}, \varphi_{i}\right) \sin m \varphi_{i}=$

$\left(\frac{2 \Phi_{D C}}{\pi}\right) \cos \left(\frac{m \varphi_{M G}}{2}\right)\left[\frac{1}{m}+\frac{m}{\left(\left(\pi / \varphi_{M G}\right)^{2}-m^{2}\right)}\right]$,

with other coefficients being zero as indicated above. The angular extent of the merging gap has been somewhat arbitrarily set as $\varphi_{M G}=45^{\circ}$, so that inflow (and outflow) of open flux into the polar cap is restricted to one quarter of the angular extent of the dayside (and nightside) boundaries. We also take $\Phi_{D C}=200 \mathrm{kV}$ as previously indicated.

The above equations thus define the potential associated with the twin-vortex flow. The electric field and flow components are then given by

$E_{i T V \theta}=-B_{i} V_{i T V \varphi}=-\frac{1}{R_{i} \sin \theta_{i}} \frac{\partial \Phi_{i T V}}{\partial x_{i}}$

and

$E_{i T V \varphi}=B_{i} V_{i T V \theta}=-\frac{1}{R_{i} \sin \theta_{i}} \frac{\partial \Phi_{i T V}}{\partial \varphi_{i}}$,

so that in the polar cap we have (in general)

$E_{i T V \theta}=-B_{i} V_{i T V \varphi}=-\frac{1}{R_{i} \sin \theta_{i}}$
$\sum_{m=1}^{\infty} m\left(a_{m} \sin m \varphi_{i}+b_{m} \cos m \varphi_{i}\right) \exp \left(m\left(x_{i}-x_{i B P C}\right)\right)$ (30a) 
and

$E_{i T V \varphi}=B_{i} V_{i T V \theta}=-\frac{1}{R_{i} \sin \theta_{i}}$

$\sum_{m=1}^{\infty} m\left(a_{m} \cos m \varphi_{i}-b_{m} \sin m \varphi_{i}\right) \exp \left(m\left(x_{i}-x_{i B P C}\right)\right)$

while in the outer magnetosphere

$E_{i T V \theta}=-B_{i} V_{i T V \varphi}=$

$$
\begin{aligned}
& \frac{1}{R_{i} \sin \theta_{i}} \sum_{m=1}^{\infty} m\left(a_{m} \sin m \varphi_{i}+b_{m} \cos m \varphi_{i}\right) \\
& \frac{\cosh \left(m\left(x_{i B O M}-x_{i}\right)\right)}{\sinh \left(m\left(x_{i B O M}-x_{i B P C}\right)\right)}
\end{aligned}
$$

and

$$
\begin{aligned}
& E_{i T V \varphi}=B_{i} V_{i} T V \theta= \\
& -\frac{1}{R_{i} \sin \theta_{i}} \sum_{m=1}^{\infty} m\left(a_{m} \cos m \varphi_{i}-b_{m} \sin m \varphi_{i}\right) \\
& \quad \frac{\sinh \left(m\left(x_{i B O M}-x_{i}\right)\right)}{\sinh \left(m\left(x_{i B O M}-x_{i B P C}\right)\right)} .
\end{aligned}
$$

\subsection{Ionospheric and field-aligned currents}

Using the electric fields and potentials of the rotational and twin-vortex flows described above, we can now determine both the horizontal ionospheric Pedersen current from Eq. (7a), and the consequent field-aligned current from Eq. (8). Hall currents are not considered, since with the assumptions given above these close wholly in the ionosphere as previously indicated. Adding the contributions from the twin-vortex and the rotational flows, we find from Eqs. (7a) and (18b) the total Pedersen current to be given by

$i_{P}=\Sigma_{P}^{*}\left(E_{i T V}+B_{i} R_{i} \sin \theta_{i}\left(\Omega_{S}-\omega_{R O}(F)\right) \hat{\theta}\right)$.

Substituting into Eq. (8) to find the field-aligned current density, we find that the term associated with the twin vortex flow drops out due to the assumption in Eq. (21) as previously indicated, such that the distributed field-aligned current density is associated only with the rotational component of the flow, given by

$j_{\| i}=-\frac{\Sigma_{P}^{*} B_{i}}{\sin \theta_{i}} \frac{d}{d \theta_{i}}\left[\sin ^{2} \theta_{i}\left(\Omega_{S}-\omega_{R O}(F)\right)\right]$.

The distributed field-aligned current in the model is thus axisymmetric. For the polar cap region we find

$j_{\| i}=-2 \Sigma_{P}^{*} B_{i}\left(\Omega_{S}-\omega_{P C}\right) \cos \theta_{i}$, while for the outer magnetosphere

$j_{|| i}=-2 \Sigma_{P}^{*} B_{i}\left(\Omega_{S}-\omega_{O M}\right) \cos \theta_{i}$.

The distributed field-aligned currents in these regions are thus directed downward into the ionosphere, vary slowly with co-latitude, and are larger in the polar cap than in the outer magnetosphere if $\omega_{O M}$ is closer to rigid corotation than is $\omega_{P C}$ as assumed here. For the middle magnetosphere we also find from Eq. (17c)

$$
\begin{aligned}
& j_{\| i}=-\Sigma_{P}^{*} B_{i}\left(\Omega_{S}-\omega_{R O}(F)\right) \\
& \left(2 \cos \theta_{i}-\frac{\sin ^{2} \theta_{i}}{\left(1-\cos \theta_{i}\right)} \frac{n}{\left(\left(F_{M M} / F\right)^{n}+1\right)}\right),
\end{aligned}
$$

where $F$ is given by Eq. (1). This function is such that the field-aligned current density is negative (downward) at the BOM boundary, then reverses to positive with increasing colatitude, before peaking and falling to small values as the plasma angular velocity approaches rigid corotation, as will be shown in the results below. These properties are essentially the same as those in the axi-symmetric model of Cowley et al. (2004b).

In addition to these distributed field-aligned currents, sheet field-aligned currents also flow at the circular BPC and BOM boundaries due to discontinuities in the co-latitudinal Pedersen currents. These discontinuities are driven by shears in the azimuthal flow across the boundaries, with contributions being provided both by the rotational and the twin-vortex flow components. The contribution from the rotational flow component is independent of local time, while the twin-vortex flow provides dawn-dusk asymmetries as discussed above. From Eqs. (17a, b) and (32), the contribution at the polar cap boundary (BPC) from the rotational flow component is

$i_{\| i R O}=\Sigma_{P}^{*} B_{i} R_{i} \sin \theta_{i B P C}\left(\omega_{O M}-\omega_{P C}\right)$,

where a positive value indicates an upward-directed current, while using Eqs. $(17 \mathrm{~b}, \mathrm{c})$ at the outer magnetosphere boundary (BOM) we have

$i_{\| i R O}=-\Sigma_{P}^{*} B_{i} R_{i} \sin \theta_{i \text { BOM }}\left(\omega_{O M}-\omega_{B M M}\right)$.

Similarly from Eqs. (30a), (31a), and (32), the contribution at the polar cap boundary from the twin vortex flow is (in general)

$$
\begin{aligned}
& i_{\| i T V}=-\frac{2 \Sigma_{P}^{*}}{R_{i} \sin \theta_{i B P C}} \\
& \sum_{m=1}^{\infty} m\left(a_{m} \sin m \varphi_{i}+b_{m} \cos m \varphi_{i}\right) \\
& \frac{1}{\left(1-\exp \left(-2 m\left(x_{i} \text { BOM }-x_{i B P C}\right)\right)\right)},
\end{aligned}
$$




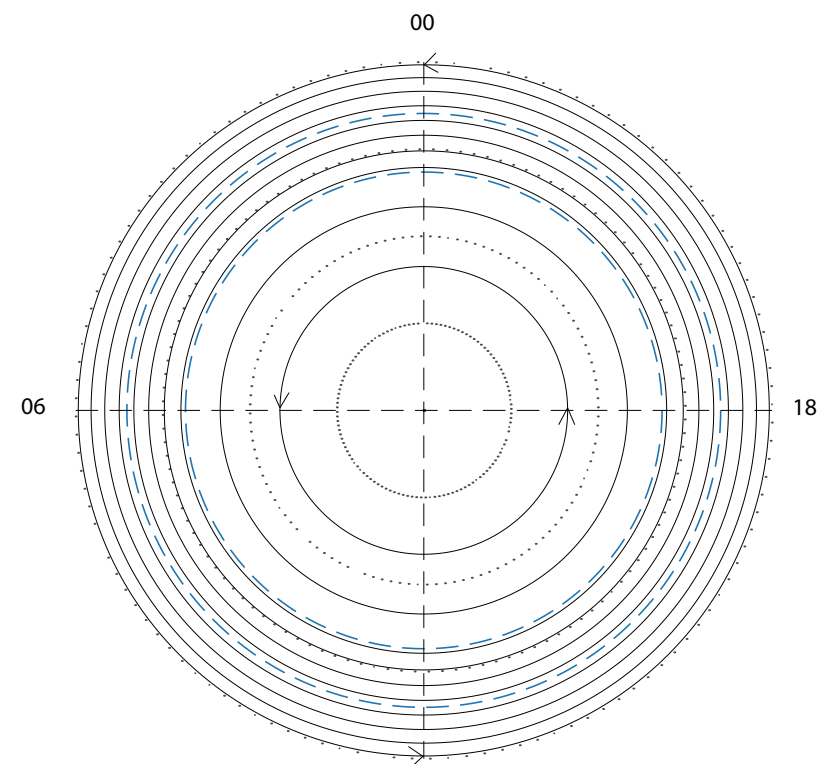

12

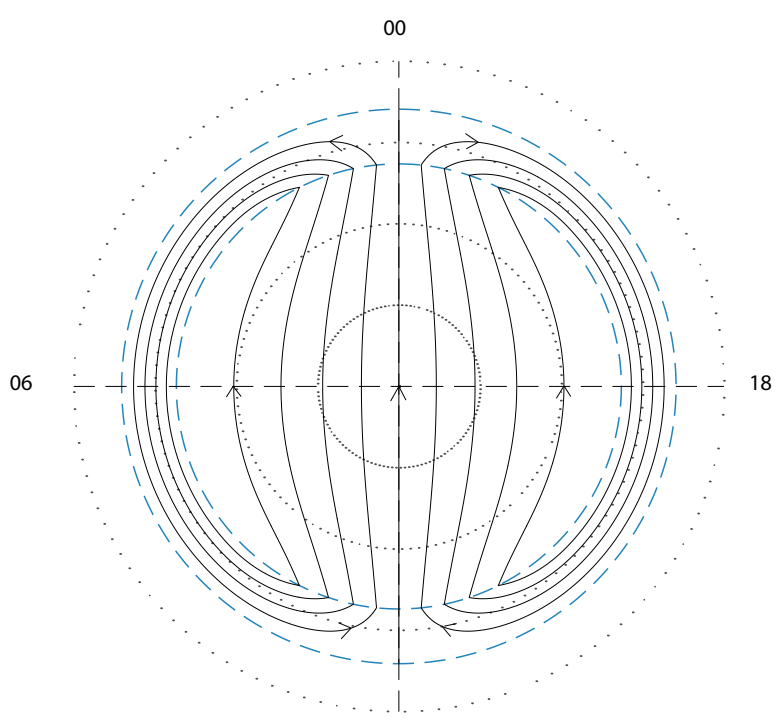

12

(b)

00

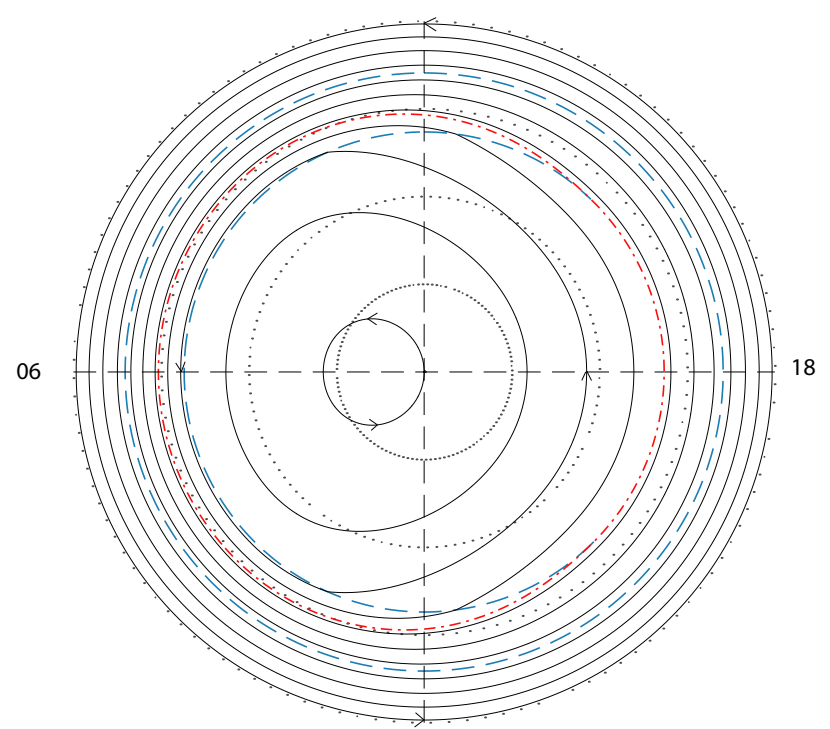

12

(c)

Fig. 2. Streamlines of the model ionospheric flow in the inertial frame, obtained by contouring the electrostatic potential, $\Phi_{i}$ on a polar grid. As in Fig. 1, the direction to the Sun is at the bottom of the diagrams, dusk to the right, and dawn to the left. The region from the pole to $20^{\circ}$ co-latitude is shown, marked at $5^{\circ}$ intervals by dotted circles. Inner and outer circles shown by the blue dashed lines indicate the boundary of the polar cap (BPC) and the boundary of the outer magnetosphere (BOM), respectively. Long dashed lines indicate the noon-midnight and dawn-dusk meridians. (a) Shows contours of the potential for the rotational flow component only, at consistent $100 \mathrm{kV}$ intervals from zero at the pole to $1000 \mathrm{kV}$ at the outermost contour at $\sim 20^{\circ}$ co-latitude. (b) Shows contours of the potential for the twin vortex flow only in a similar format, except that contours are shown at intervals of $20 \mathrm{kV}$. (c) Shows contours of potential for the combined rotational and twin vortex flow (i.e. the potentials shown in (a) and (b) added together), with contours of potential again being shown at $100 \mathrm{kV}$ intervals. The red dot-dashed line marks the boundary between the regions of Dungey-cycle and Vasyliunas-cycle flow in the outer magnetosphere, at a potential of $\sim 373.6 \mathrm{kV}$. 
while at the outer magnetosphere boundary we have from Eq. (31a) and recalling that the twin vortex flow is taken to be zero in the middle magnetosphere

$$
\begin{gathered}
i_{\| i T V}=\frac{2 \Sigma_{P}^{*}}{R_{i} \sin \theta_{i \text { ВOM }}} \\
\sum_{m=1}^{\infty} m\left(a_{m} \sin m \varphi_{i}+b_{m} \cos m \varphi_{i}\right) \\
\frac{1}{\sinh \left(m\left(x_{i} \text { ВOM }-x_{\text {B } B C C}\right)\right)} .
\end{gathered}
$$

This completes the mathematical description of our model of the flows and currents in Saturn's polar ionosphere. We now proceed to present the results derived on this basis.

\section{Results}

\subsection{Flow streamlines}

We begin our discussion by presenting an overview of the overall pattern of ionospheric plasma flow in our model. This is illustrated in Fig. 2, where we show the streamlines of the flow in polar plots, obtained by contouring the associated electrostatic potential, $\Phi_{i}$. In Figs. 2a-c we show separately the streamlines of the rotational flow, the twin vortex flow, and the combined flow, respectively. In each plot the contours are shown at fixed voltage intervals, such that their spacing gives an impression of the relative speed of the flow in the various model regions. As in Fig. 1, the direction toward the Sun (i.e. noon, corresponding to $\varphi_{i}=0^{\circ}$ ) is at the bottom of each diagram, dusk $\left(\varphi_{i}=90^{\circ}\right)$ is to the right, and dawn $\left(\varphi_{i}=270^{\circ}\right)$ to the left. The region from the pole to $20^{\circ}$ co-latitude is shown, marked at $5^{\circ}$ intervals by the dotted circles. The circles shown by the blue dashed lines then indicate the "polar cap boundary" (BPC), the interface between the open flux region and the outer magnetosphere, and the "outer magnetosphere boundary" (BOM), the interface between the outer and middle magnetosphere, located at $\sim 13.68^{\circ}$ and $\sim 17.05^{\circ}$ co-latitude, respectively.

Figure 2a shows a plot of the streamlines for the eastwarddirected rotational flow only, given by Eqs. (17)-(20). The contours of potential in this case are shown at $100 \mathrm{kV}$ intervals, from zero at the pole at the centre of the plot, to $1000 \mathrm{kV}$ at the outermost contour shown. It can be seen that the potential increases relatively slowly within the polar cap region of open field lines, indicative of the relatively slow rotation of the plasma, reaching $\sim 274 \mathrm{kV}$ at the polar cap boundary. The azimuthal flow then increases in the outer magnetosphere ring, as indicated by the more closely-spaced streamlines in this region, such that the potential rises to $\sim 649 \mathrm{kV}$ at the outer magnetosphere boundary. Beyond this, in the middle magnetosphere, the azimuthal velocities initially fall somewhat, before increasing again at lower latitudes as the plasma angular velocity increases towards near-rigid corotation with the planet.

Figure $2 \mathrm{~b}$ shows a plot of the flow streamlines for the twin vortex flow only, given by Eqs. (23), (24) and (27). This plot has been derived using 25 terms in the Fourier series in Eqs. (23) and (24) (from $m=1$ to $m=51$ with $m$ odd only), as is the case for all the plots shown in this section. The format is the same as for Fig. 2a, except that the spacing between the contours has been reduced to $20 \mathrm{kV}$. It can be seen that the twin vortex provides a roughly uniform antisunward flow across the polar cap, though with somewhat higher speeds near the boundary at noon and midnight, compared with dawn and dusk, due to the confined "merging gap" nature of the flow across the open-closed field line boundary. These streamlines then close wholly in the outer magnetosphere ring at lower latitudes, symmetrically between dawn and dusk. The potential contours are much more closely spaced in this region, indicating faster flows directed almost azimuthally via dawn and dusk. The twin vortex flow terminates at the outer magnetosphere boundary (outer blue longdashed circle), and does not extend into the middle magnetosphere region at lower latitudes.

The streamlines of the combined flow, obtained by adding the potentials contoured in Figs. $2 a$ and $2 b$, are shown in Fig. 2c, where the streamlines are again shown with $100 \mathrm{kV}$ between each contour, as in Fig. 2a. It can be seen that two types of streamline are now present in the region of open field lines, reflecting the contrasting behaviours in Figs. $2 \mathrm{a}$ and $2 \mathrm{~b}$. On the dusk side of the polar cap, streamlines pass between the dayside and nightside "merging gaps", corresponding to an anti-sunward motion driven by the solar wind, combined with a strongly sub-corotational circulation. These streamlines are then closed in the outer magnetosphere ring via dawn, within the region bounded by the red dot-dashed line. The red line thus marks the boundary between the Dungeycycle flow in the polar region and the Vasyliunas-cycle flow at lower latitudes (like the dot-dashed line in Fig. 1b), and is at a potential of $\sim 373.6 \mathrm{kV}$. On the dawn side of the polar cap, however, streamlines close wholly within the region of open field lines. Although the existence of closed flow streamlines contained within the polar cap may initially seem counterintuitive, Milan et al. (2005) have recently discussed how the plasma rotation within the polar cap promotes the closure of more recently opened field lines in the tail, as implied by Fig. 2c, while others may remain within the tail for longer intervals, thus forming a very extended tail of connected open field lines. Eventually, however, such regions of open flux will become closed again, during episodes of strong tail reconnection that reach to very high latitudes (e.g. Cowley et al., 2005a).

The overall dawn-dusk asymmetry effects introduced by adding the twin vortex flow to the rotational flow (the latter being essentially the flow in the axi-symmetric model of Cowley et al., 2004b), can thus be seen qualitatively by comparison of Figs. 2a and c. In the polar cap, the twin vortex 


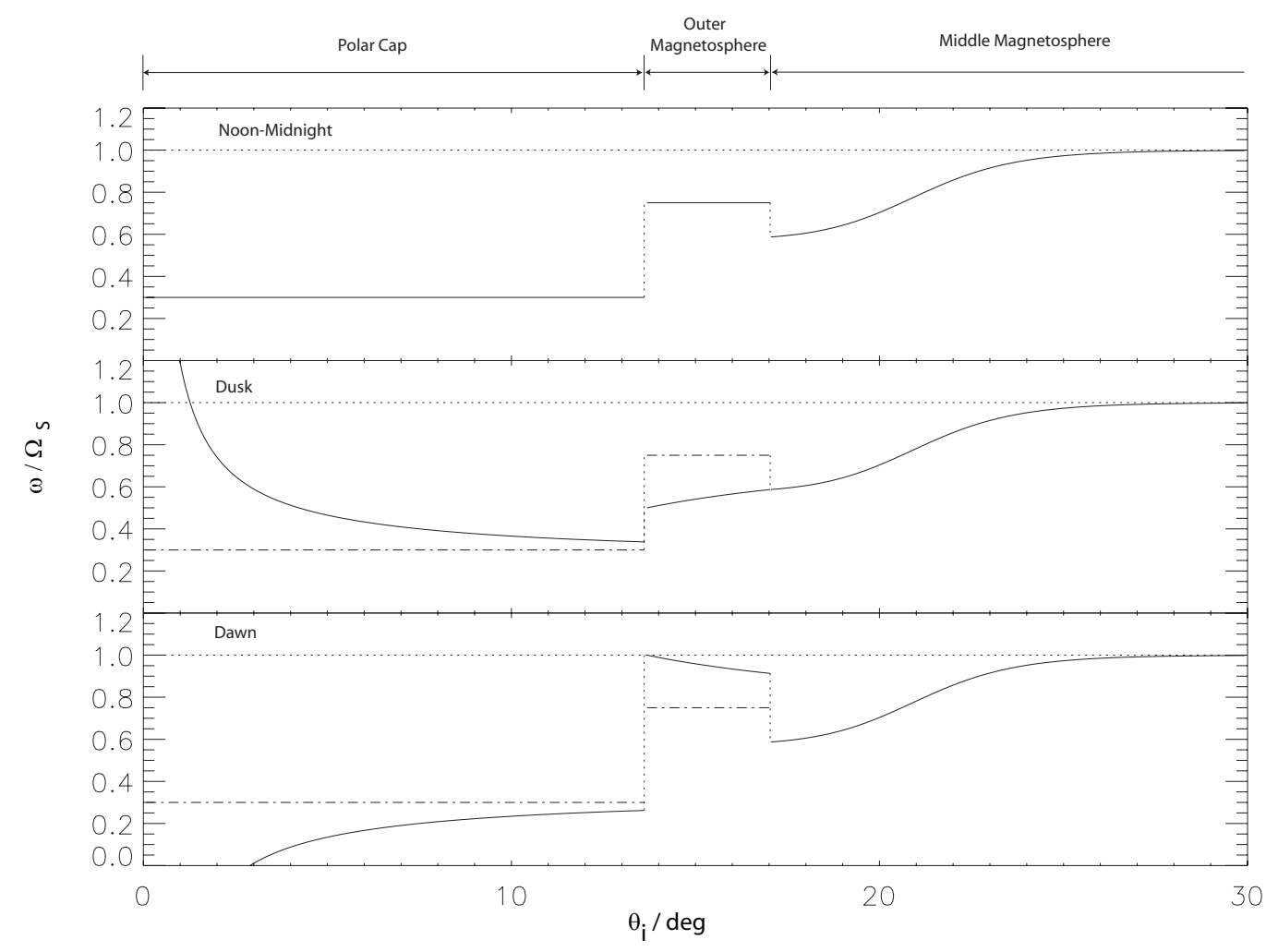

Fig. 3. Plot of the total plasma angular velocity in the model, $\omega$, defined by Eq. (37b), normalized to Saturn's angular velocity, $\Omega_{S}$, shown versus co-latitude angle $\theta_{i}$ on the principal meridians. The horizontal bars at the top of the figure indicate the regions of co-latitude that map to the polar cap, the outer magnetosphere, and the middle magnetosphere regions. The solid line in the upper panel shows the plasma angular velocity on the noon and midnight meridians, which results from the rotational flow component only. The horizontal dotted line shows rigid corotation. The middle panel similarly shows the total plasma angular velocity on the dusk meridian, shown by the solid line, while for comparison the dot-dashed line shows the rotational flow component only, as on the noon-midnight meridian in the upper panel. The solid line in the lower panel then shows the total plasma angular velocity on the dawn meridian, while the dot-dashed line again shows the rotational flow component only.

flow reduces the eastward flow at dawn, and enhances it at dusk. In the outer magnetosphere region, on the other hand, the twin vortex flow enhances the eastward flow at dawn (where the flow corresponds to the sum of the Vasyliunas cycle and the Dungey-cycle return flow), and reduces it at dusk (where the flow corresponds to the Vasyliunas cycle alone). Consequently, the addition of the twin vortex enhances the flow shears at both the polar cap and outer magnetosphere boundaries at dawn, and hence also the sheet field-aligned currents, while reducing them at both boundaries at dusk. In the following sections we now examine these asymmetry effects more quantitatively.

\subsection{Plasma velocities}

In Figs. 3 and 4 we show co-latitude profiles of the azimuthal flow from the pole to $30^{\circ}$ co-latitude on the principal local time meridians, in two complementary formats. In Fig. 3 we show the plasma angular velocity $\omega$ of the combined flow normalized to $\Omega_{S}$, where $\omega$ is defined simply by

$$
\omega=\frac{V_{i \varphi}}{R_{i} \sin \theta_{i}},
$$

where $V_{i \varphi}$ is the total plasma azimuthal velocity. This format lends itself to discussion of departures of the plasma flow from rigid corotation. In Fig. 4 we show the total azimuthal velocity, which is proportional to the ionospheric electric field, and is directly related to the ionospheric currents. The upper panel in each figure shows the azimuthal plasma flow on the noon and midnight meridians, where the twin vortex flow makes no contribution (its velocities are purely meridional at these local times). These flows therefore correspond to the rotational flow component only, similar to the axi-symmetric model of Cowley et al. (2004b). The two lower panels then show the asymmetries at dusk and dawn introduced in the model developed here.

Considering first the noon-midnight meridian in the upper panels, the plasma angular velocity in Fig. 3 remains at a fixed value of $0.3 \Omega_{S}$ within the polar cap region, while 


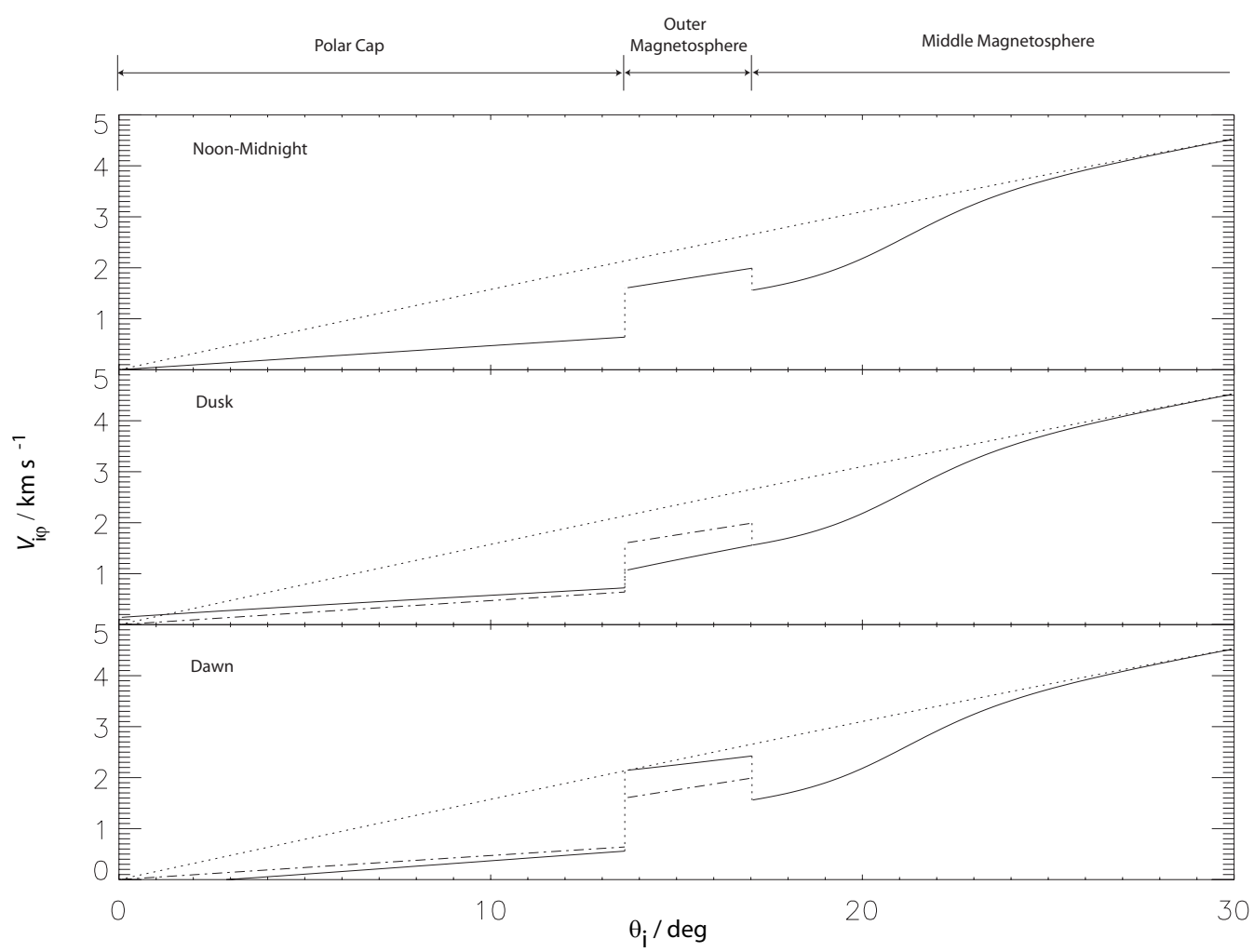

Fig. 4. Plot of the total plasma azimuthal velocity versus co-latitude angle $\theta_{i}$ on the principal meridians, given by the sum of the twin vortex flow component $V_{i} T V \varphi$ from Eqs. (30a) and (31a), and the rotational flow component $V_{i} R O$ in Eq. (18a). The format follows that of Fig. 3. The solid line in the upper panel thus shows the azimuthal velocity in $\mathrm{km} \mathrm{s}^{-1}$ on the noon and midnight meridians, given by the rotational flow component only, while the long dotted line shows the azimuthal velocity corresponding to rigid corotation with the planet (as in all panels of the figure). The middle panel then shows the total azimuthal velocity profile on the dusk meridian in a similar format, where the dot-dashed line shows for comparison the contribution of the rotational component only, as in the upper panel. The lower panel then shows the azimuthal velocity on the dawn meridian in the same format.

increasing to $0.75 \Omega_{S}$ in the outer magnetosphere. It then falls to a local minimum of $\sim 0.59 \Omega_{S}$ in the middle magnetosphere region, before increasing once more to approach near-rigid corotation (horizontal dotted line) at $\sim 30^{\circ}$ colatitude. The corresponding plasma azimuthal velocities in Fig. 4 show an approximately linear increase in the polar cap, with a jump from $\sim 0.64 \mathrm{~km} \mathrm{~s}^{-1}$ to $\sim 1.61 \mathrm{~km} \mathrm{~s}^{-1}$ at the polar cap boundary. The velocity then increases again in the outer magnetosphere region before falling from $\sim 1.99 \mathrm{~km} \mathrm{~s}^{-1}$ to a local minimum of $\sim 1.56 \mathrm{~km} \mathrm{~s}^{-1}$ across the boundary of the outer magnetosphere. The azimuthal velocity profile then increases once more, to $\sim 4.52 \mathrm{~km} \mathrm{~s}^{-1}$ in the near-rigidly corotating region at $30^{\circ}$ co-latitude. The dotted line in this panel (and those below) again indicates the azimuthal velocity for rigid corotation.

The middle panels of Figs. 3 and 4 then show the azimuthal flows on the dusk meridian, where the dot-dashed lines correspond to the rotational flow component only, as in the upper panels, for purposes of comparison. Here, the effect of the twin vortex is to increase the plasma angular velocity within the polar cap region, to $\sim 0.34 \Omega_{S}$ at the polar cap boundary (the singularity at the pole itself is simply due to the finite anti-sunward flow of the twin vortex at this point). However, the effect in the outer magnetosphere region is to reduce the angular velocity to $\sim 0.50 \Omega_{S}$ at its poleward edge, such that the jump in angular velocity across the polar cap boundary is now strongly reduced compared with that on the noon-midnight meridian shown in the upper panel of Fig. 3. The jump in the azimuthal velocity at the polar cap boundary in Fig. 4 is similarly reduced, now increasing from $\sim 0.72 \mathrm{~km} \mathrm{~s}^{-1}$ in the polar cap to $\sim 1.07 \mathrm{~km} \mathrm{~s}^{-1}$ in the outer magnetosphere. Beyond the polar cap boundary the angular velocity (and the azimuthal velocity) then monotonically increase with co-latitude, the velocity step at the outer magnetosphere boundary being (by design) reduced to zero at this meridian.

The lower panels of Figs. 3 and 4 similarly show the effects on the dawn meridian. It can be seen that the effect of the twin vortex is now such as to decrease the plasma angular velocity in the polar cap region, while increasing it in 


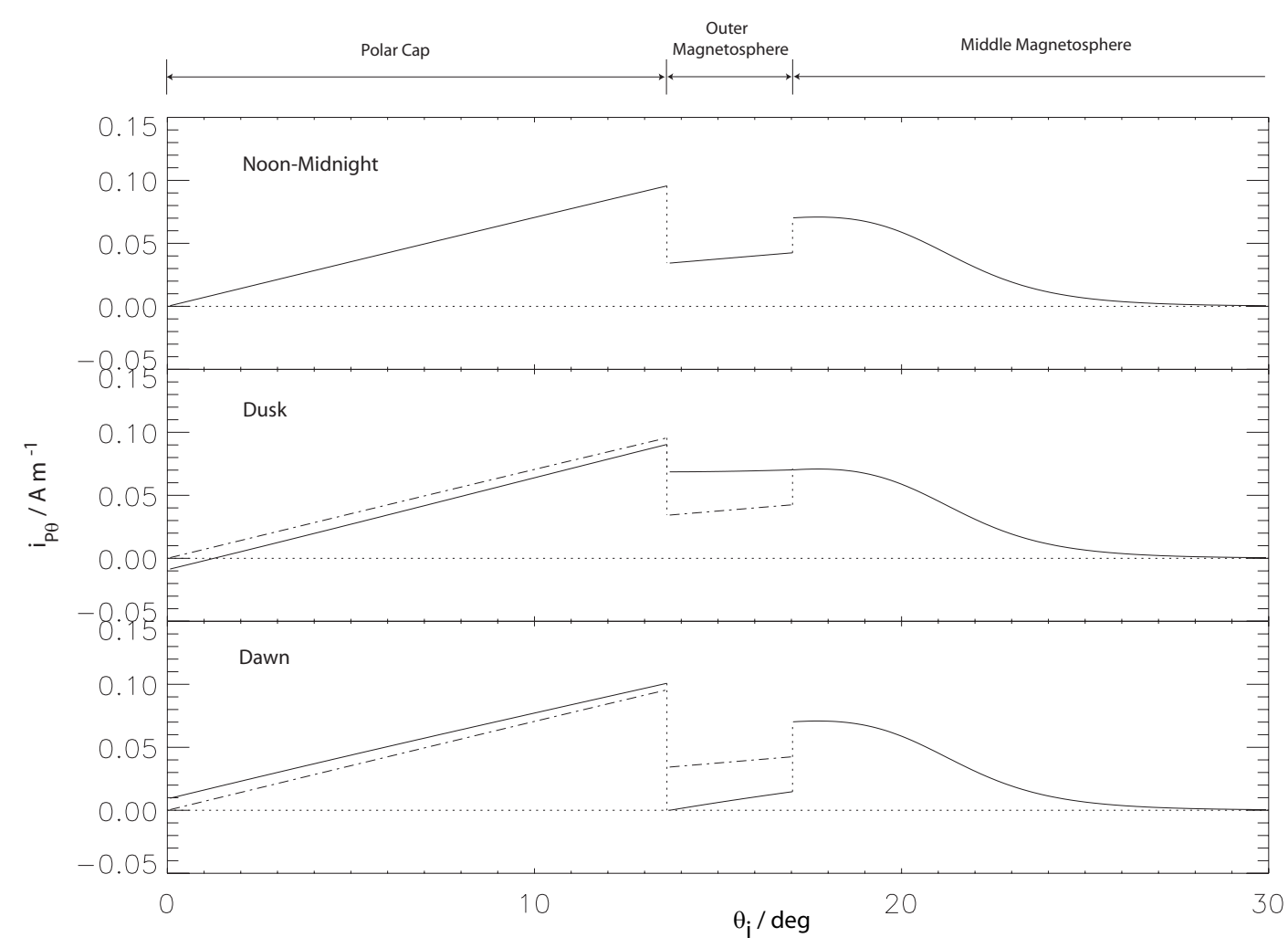

Fig. 5. Plot of the co-latitudinal component of the horizontal ionospheric Pedersen current intensity, $i_{P \theta}$ given by Eq. (32), shown versus colatitude angle $\theta_{i}$ on the principal meridians, assuming $\Sigma_{P}^{*}=1$ mho. The format follows that of Figs. 3 and 4 . The solid line in the upper panel thus shows the Pedersen current intensity in $\mathrm{A} \mathrm{m}^{-1}$ on the noon and midnight meridians, associated with the rotational flow component only. The horizontal dotted line indicates the zero level (the current for rigid corotation). The solid line in the middle panel shows the co-latitudinal component of the Pedersen current intensity on the dusk meridian while the dot-dashed line shows for comparison the contribution of the rotational component only, as in the upper panel. The lower panel shows the co-latitudinal component of the Pedersen current intensity on the dawn meridian, in the same format.

the outer magnetosphere region. The effect is thus to enhance the jumps in flow at both boundaries. At the polar cap boundary the angular velocity increases from $\sim 0.26 \Omega_{S}$ in the polar cap to approximately rigid corotation in the outer magnetosphere. This corresponds to an increase in azimuthal velocity in the lower panel of Fig. 4 from $\sim 0.56 \mathrm{~km} \mathrm{~s}^{-1}$ to $\sim 2.15 \mathrm{~km} \mathrm{~s}^{-1}$. At the outer magnetosphere boundary the angular velocity then falls from $\sim 0.91 \Omega_{S}$ to $\sim 0.58 \Omega_{S}$, corresponding to a fall in azimuthal velocity from $\sim 2.43 \mathrm{~km} \mathrm{~s}^{-1}$ to $\sim 1.56 \mathrm{~km} \mathrm{~s}^{-1}$. The flow shears at the dawn boundary are thus enhanced compared with the rotational flow alone on the noon and midnight meridians.

\subsection{Ionospheric Pedersen currents}

The horizontal ionospheric Pedersen current intensity, given by Eq. (32), is directly proportional to the electric field in the rigidly corotating frame (through use of the effective ionospheric Pedersen conductance), and hence to the plasma velocity in this frame. The principal meridional (equatorward-directed) component of the current, which can be written as $i_{P \theta}=\Sigma_{P}^{*} B_{i}\left(R_{i} \Omega_{S} \sin \theta_{i}-V_{i \varphi}\right)$, is thus proportional to the displacement of the azimuthal velocity curves shown in Fig. 4 from the curve for rigid corotation (light dotted lines). Co-latitude profiles of the currents on the principal meridians are shown in Fig. 5, in a similar format to Figs. 3 and 4, for an effective ionospheric Pedersen conductance of 1 mho. The current can then be simply scaled for any other choice of conductivity value.

The upper panel of the figure again shows the meridional Pedersen current on the noon and midnight meridians, where the corresponding azimuthal flow is due just to the rotational flow component. This current profile is thus again essentially similar to the axi-symmetric model of Cowley et al. (2004b). Within the polar cap, the equatorward current increases near-linearly with distance from the pole, peaking at $\sim 0.096 \mathrm{~A} \mathrm{~m}^{-1}$ at the polar cap boundary. This nearlinear rise in the current intensity occurs where the model angular velocity is constant at $0.3 \Omega_{S}$, such that the azimuthal plasma velocity in the rigidly corotating frame increases near-linearly with distance from the pole as seen 


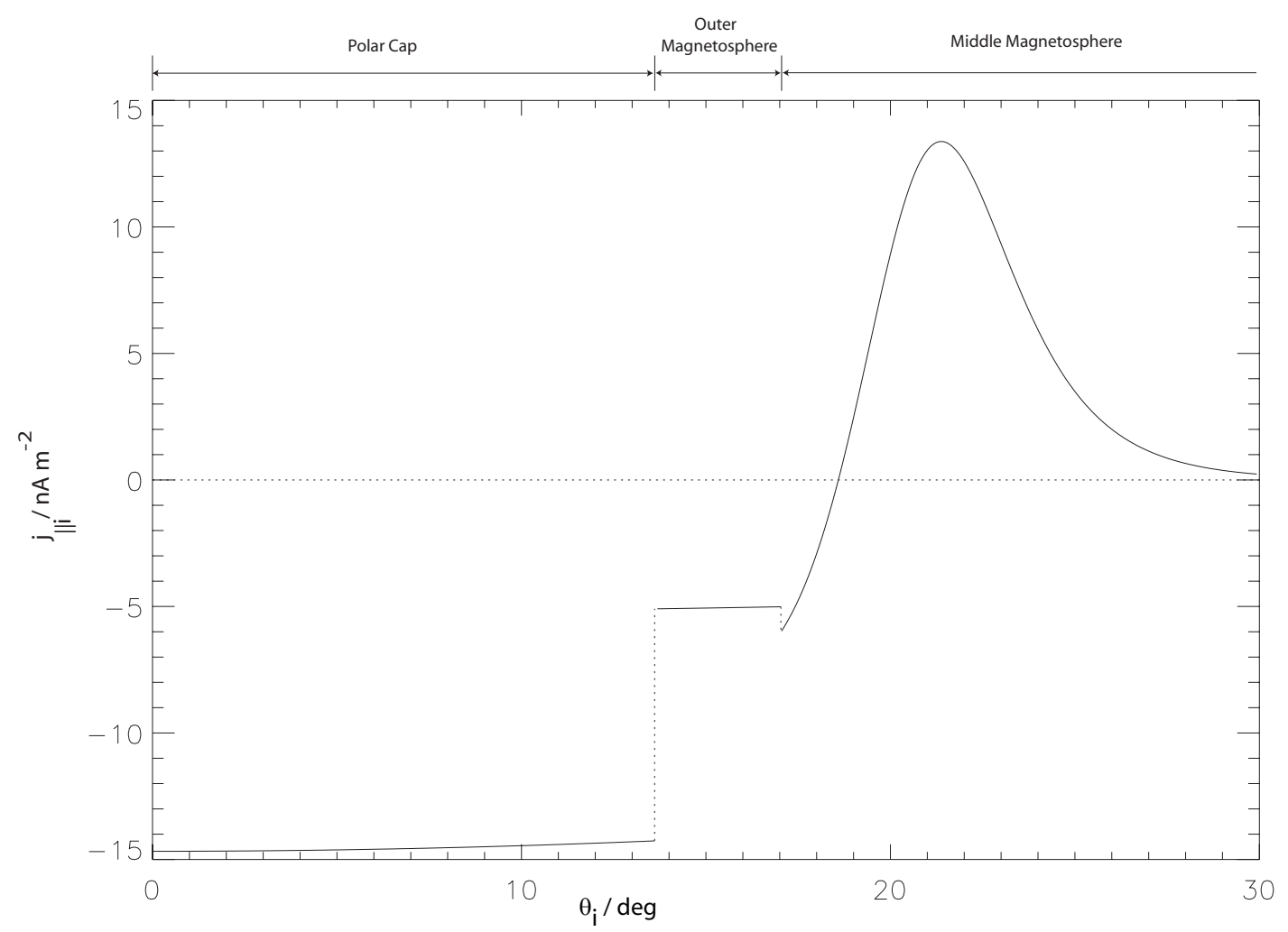

Fig. 6. Plot of the distributed field-aligned current density $j_{\| i}$ obtained from Eq. (34) from the rotational flow component only, shown versus co-latitude $\theta_{i}$. The horizontal bars at the top of the plot indicate the regions of co-latitude that map to the polar cap, the outer magnetosphere, and the middle magnetosphere regions. Positive values indicate currents directed upward out of the ionosphere, negative values indicate currents directed downward into the ionosphere, and the horizontal dotted line indicates the zero level.

in Fig. 4. The current then falls by a factor of $\sim 3$ to $\sim 0.034 \mathrm{~A} \mathrm{~m}^{-1}$ in the outer magnetosphere, before slowly growing to $\sim 0.043 \mathrm{~A} \mathrm{~m}^{-1}$ at the equatorward boundary of this region. The current then jumps to a local maximum of $\sim 0.070 \mathrm{~A} \mathrm{~m}^{-1}$ in the middle magnetosphere as the plasma velocity drops once more, before falling to small values at large co-latitudes as the plasma approaches near-rigid corotation. We note that the growth in equatorward current with co-latitude in both the polar cap and outer magnetosphere requires distributed downward-directed field-aligned currents to flow in these regions, given by Eqs. (33) and (34), while the decreasing current in the middle magnetosphere requires (mainly) an upward-directed field-aligned current in this region. Similarly, the jumps in current at the polar cap and outer magnetosphere boundaries require an upward-directed sheet current at the polar cap boundary, and a downwarddirected sheet current at the outer magnetosphere boundary, as given by Eqs. (35) and (36). These field-aligned currents will be discussed in the following section.

The equatorward Pedersen current on the dusk meridian is then shown in the middle panel of Fig. 5. The dot-dashed line represents the contribution of the rotational flow only, as shown in the upper panel, for purposes of comparison.
The current in the polar cap is now somewhat reduced compared with noon and midnight, and peaks at $\sim 0.090 \mathrm{~A} \mathrm{~m}^{-1}$ at the polar cap boundary. The current in the outer magnetosphere on the other side of the boundary, however, is increased to $\sim 0.068 \mathrm{~A} \mathrm{~m}^{-1}$. Consequently, the jump in current at the boundary is now strongly reduced compared with noon and midnight, and hence also the implied magnitude of the upward-directed sheet field-aligned current at the boundary. Beyond this boundary the Pedersen current intensity then remains almost constant in value, before falling in the middle magnetosphere as in the upper panel. There is no jump in current (or azimuthal velocity) at the outer magnetosphere boundary in this case, hence the magnitude of the downwarddirected sheet field-aligned current at the boundary is locally zero at this meridian.

The lower panel in Fig. 5 shows the equatorward-directed Pedersen current at dawn. In this case, the current in the polar cap is somewhat increased compared with noon and midnight, and peaks at $\sim 0.101 \mathrm{~A} \mathrm{~m}^{-1}$ at the polar cap boundary, before falling to near-zero in the outer magnetosphere, where the plasma near-rigidly corotates, as seen in Fig. 3. The jump in current at the polar cap boundary is thus significantly increased in this case compared with noon and midnight, and 


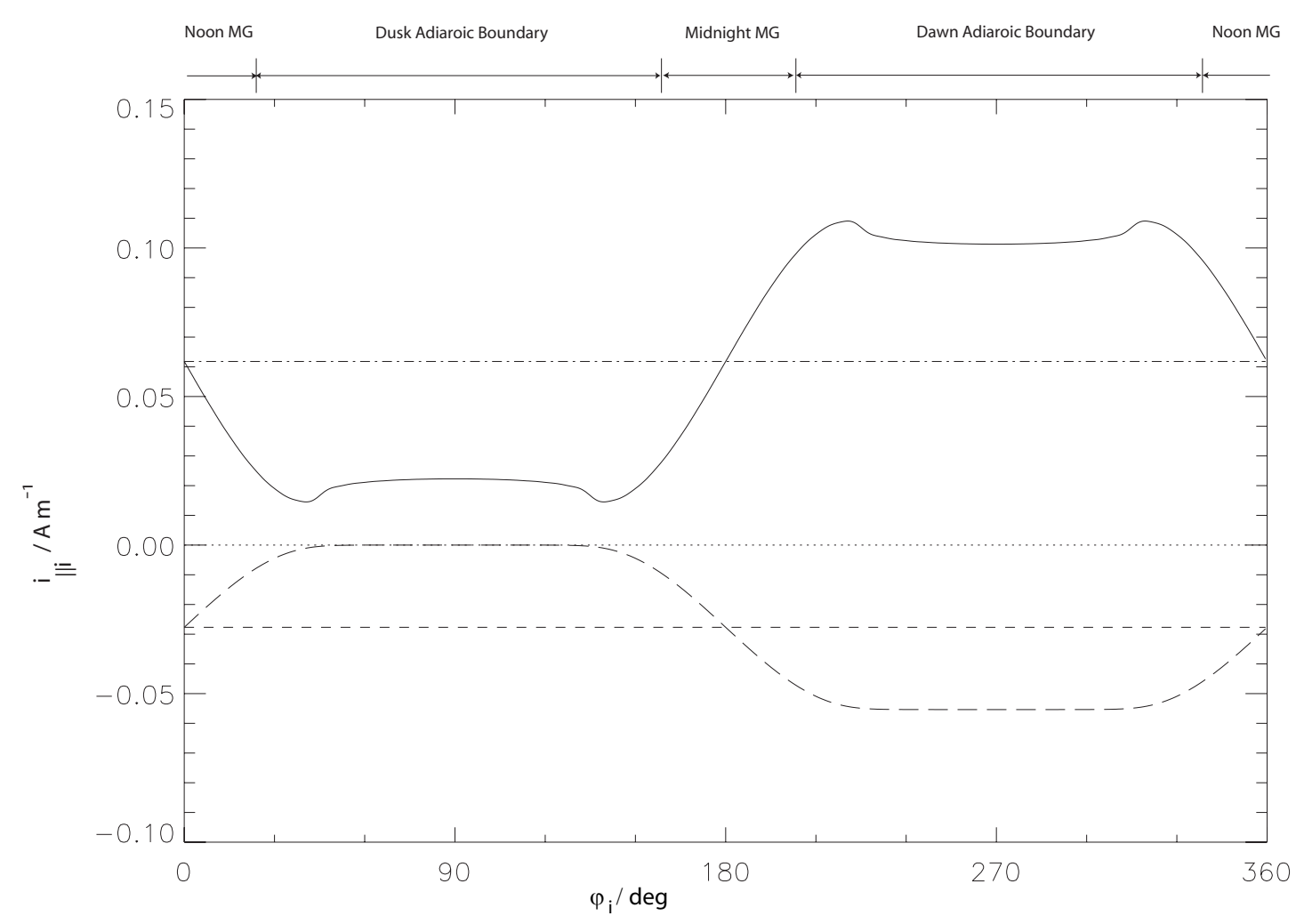

Fig. 7. Plot of the sheet field-aligned current intensities $i_{\| i}$ flowing on the polar cap and outer magnetosphere boundaries, obtained from Eqs. (35) and (36), shown versus azimuth angle $\varphi_{i}$. The solid line shows the total sheet current intensity flowing on the polar cap boundary (BPC), while the dot-dashed line shows the axi-symmetric current of the rotational flow component only. The long dashed line similarly shows the total sheet current intensity flowing on the outer magnetosphere (BOM) boundary, while the short-dashed line shows the axisymmetric current of the rotational flow component only. By definition, azimuthal angle $\varphi_{i}=0^{\circ}$ corresponds to noon, while $90^{\circ}$ corresponds to dusk, $180^{\circ}$ to midnight, and $270^{\circ}$ to dawn. The sections of the polar cap boundary corresponding to the "merging gaps" (MG) and the adiaroic sections are indicated by the horizontal bar at the top of the plot. Positive values indicate currents directed upward out of the ionosphere, negative values indicate currents directed downward into the ionosphere, and the horizontal dotted line indicates the zero level.

with it the intensity of the sheet field-aligned current at the boundary. At the outer magnetosphere boundary the current then increases from $\sim 0.015 \mathrm{~A} \mathrm{~m}^{-1}$ in the outer magnetosphere to $\sim 0.07 \mathrm{~A} \mathrm{~m}^{-1}$ in the middle magnetosphere, thus also implying a significantly increased downward sheet current at this boundary compared with noon and midnight.

\subsection{Field-aligned currents}

We now discuss the field-aligned currents flowing above the ionosphere which are required by the divergence of the horizontal ionospheric Pedersen current shown in Fig. 5. In Fig. 6 we begin by showing a co-latitude profile of the distributed field-aligned currents, given by Eqs. (33) and (34) (for a conductance of $1 \mathrm{mho}$ ), which we noted above are related to the rotational flow component only, and are thus independent of local time (azimuth) in the model. Away from the region boundaries, these currents are thus essentially similar to the field-aligned currents in the axi-symmetric model of Cowley et al. (2004b). Positive values indicate upward- directed currents, and negative values downward-directed currents. As indicated above, the growth of the Pedersen currents with co-latitude in the polar cap implies distributed downward-directed field-aligned currents in this region, with a nearly constant magnitude of $\sim 15 \mathrm{nA} \mathrm{m}^{-2}$. Similarly, in the outer magnetosphere, the currents are again downwarddirected with a reduced magnitude of $\sim 5 \mathrm{nA} \mathrm{m}^{-2}$. The decrease of the Pedersen currents in the middle magnetosphere, however, leads to upward directed field-aligned currents in this region. There is a small region of downward-directed current near the poleward boundary of the middle magnetosphere, but the current subsequently reverses sense and peaks at $\sim 13 \mathrm{nA} \mathrm{m}^{-2}$ at $\sim 21.5^{\circ}$ co-latitude, before falling to small magnitudes again at lower latitudes. These values are thus very comparable to those of the Cowley et al. (2004b) model. What is not shown in this plot, however, are the sheet fieldaligned currents on the region boundaries, directed upward at the polar cap boundary, and downward at the outer magnetosphere boundary. In the Cowley et al. (2004b) model, these sheet currents were resolved into structures of finite width, 


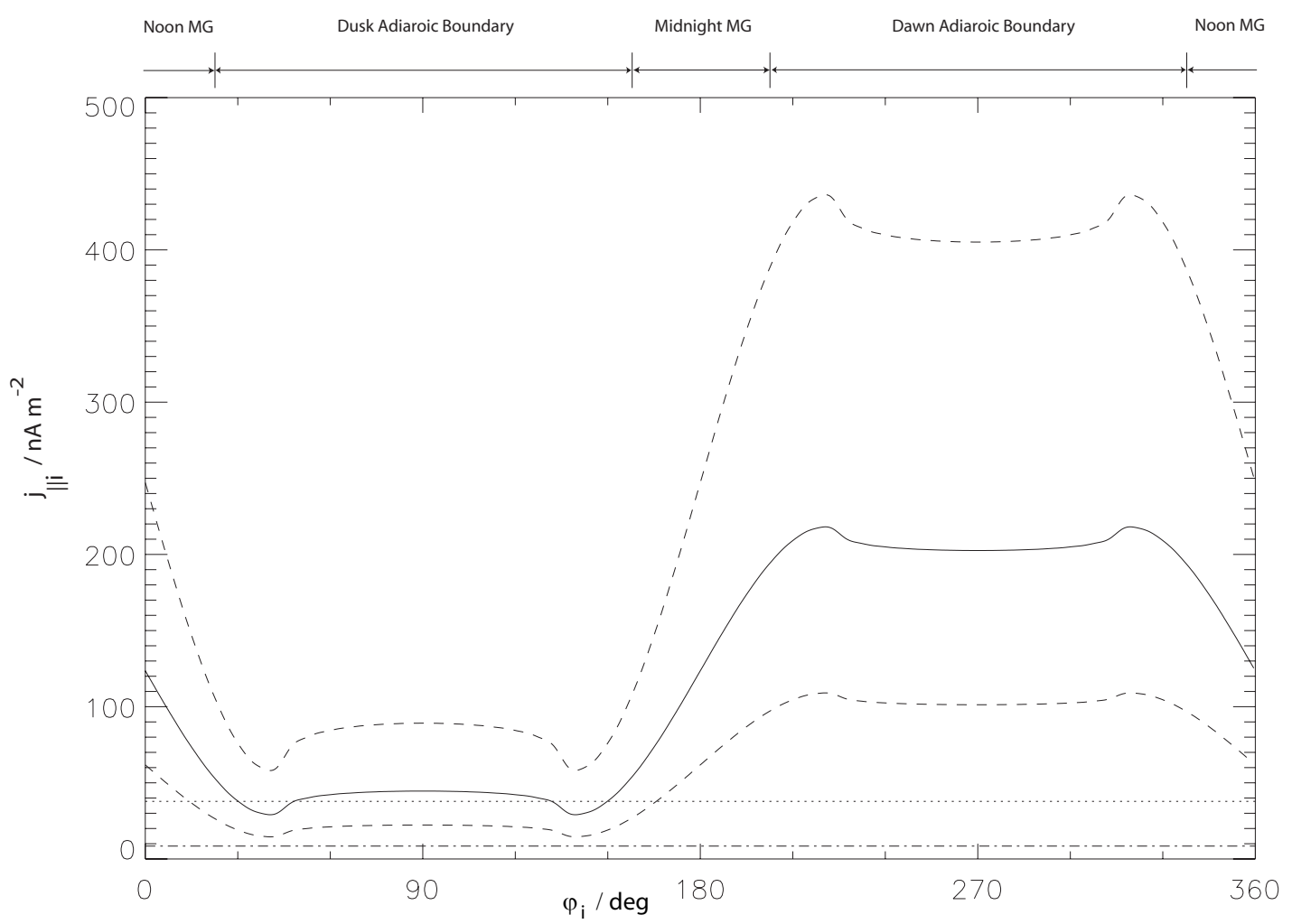

Fig. 8. Plot of the field-aligned current density flowing on the polar cap boundary for three assumed values of the boundary width, shown versus azimuth angle $\varphi_{i}$. The upper and lower dashed lines correspond to widths of 250 and $1000 \mathrm{~km}$ respectively, while the central solid line is for a width of $500 \mathrm{~km}$. The horizontal dotted line shows the limiting current density that can be carried by unaccelerated cool magnetosheath electrons, given by Eq. (9) with $N=0.2 \mathrm{~cm}^{-3}$ and $W_{t h}=50 \mathrm{eV}$, while the dot-dashed line similarly shows the limiting current density that can be carried by unaccelerated outer magnetosphere electrons with $N=0.01 \mathrm{~cm}^{-3}$ and $W_{t h}=1 \mathrm{keV}$. When the field-aligned current density exceeds the limiting value, field-aligned acceleration of auroral electrons is required.

since the angular velocity model was smoothly-varying with latitude and did not have sharp jumps as in the present case.

In Fig. 7 we thus show the intensity of the sheet fieldaligned currents on the polar cap and outer magnetosphere boundaries, plotted versus azimuth angle $\varphi_{i}$. As indicated above, $\varphi_{i}=0^{\circ}$ corresponds to noon, $\varphi_{i}=90^{\circ}$ to dusk, $\varphi_{i}=180^{\circ}$ to midnight, and $\varphi_{i}=270^{\circ}$ to dawn. The solid line shows the total sheet current flowing on the polar cap boundary, where the dot-dashed line shows the axi-symmetric current associated with the rotational flow alone. A strong local time modulation in the current is evident, with upward currents of $\sim 0.10 \mathrm{~A} \mathrm{~m}^{-1}$ at dawn, compared with $\sim 0.022 \mathrm{~A} \mathrm{~m}^{-1}$ at dusk, in conformity with the above discussion. The switch between these values across noon and midnight occurs in the region of the twin vortex "merging gaps", as indicated at the top of the figure, with localized maxima and minima in the current intensity occurring near their "ends" due to a localised maximum in the flow vorticity in these regions. The long dashed line similarly shows the total sheet current flowing on the outer magnetosphere boundary, with the short dashed line again showing the value associated with the rota- tional flow alone. The downward-directed current intensity is near-constant at $\sim-0.055 \mathrm{~A} \mathrm{~m}^{-1}$ on the portion of the boundary around dawn, but decreases to near-zero on the corresponding portion of the boundary at dusk, in conformity with the above discussion of Figs. 4 and 5.

\subsection{Auroral parameters}

We now consider the implications of the results concerning the upward-directed field-aligned currents for electron acceleration and aurorae. We recall from Sect. 3.3 that fieldaligned acceleration of magnetospheric electrons is required if the upward-directed field-aligned current density exceeds the maximum value that can be provided by the unaccelerated magnetospheric electron population, given by Eq. (9), which depends on the density and temperature of the source plasma.

We first briefly consider the distributed upward fieldaligned current in the middle magnetosphere region, shown in Fig. 6. The radial dependence of the density and temperature of the source plasma within the middle magnetosphere has been determined by Richardson $(1986,1995)$ 


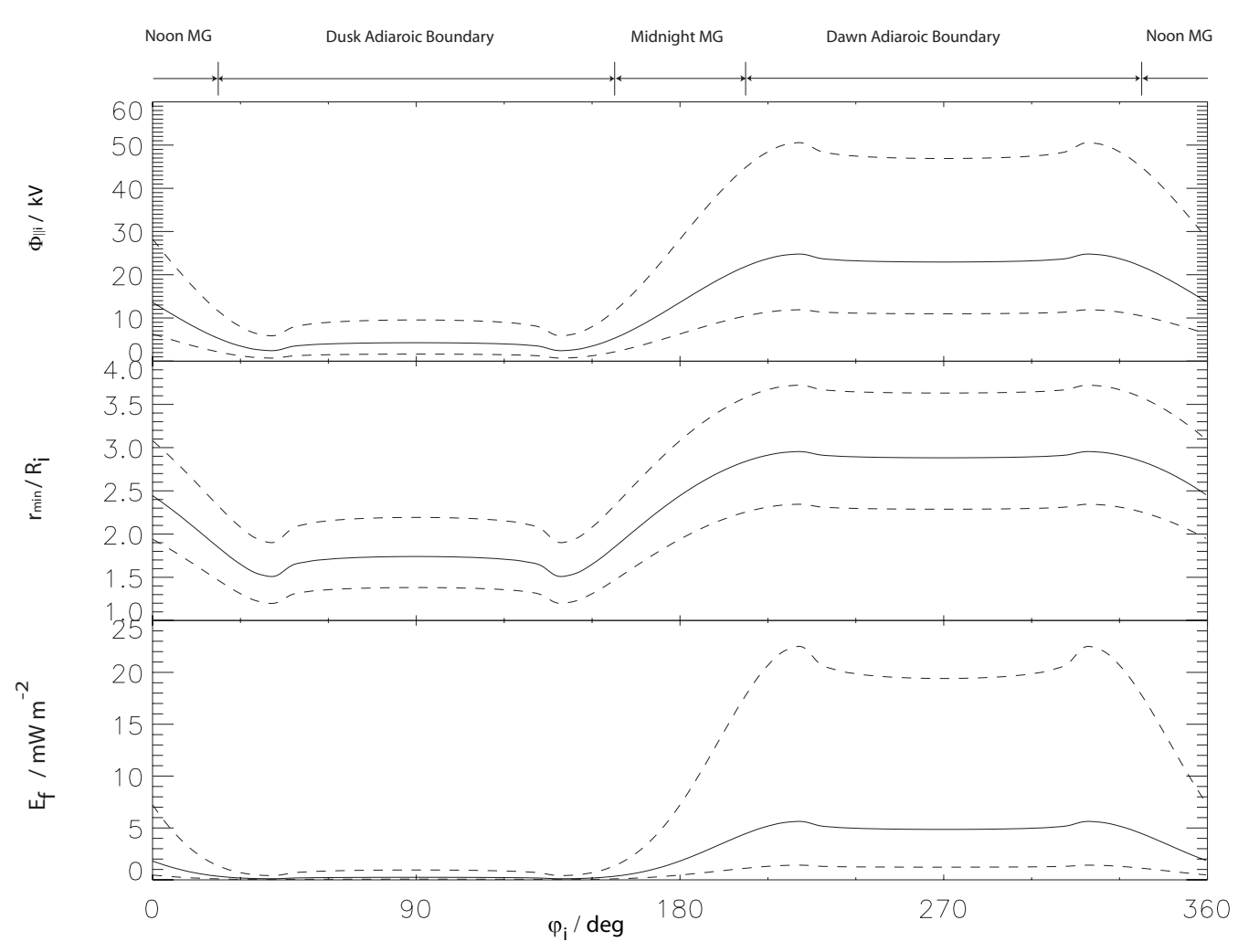

Fig. 9a. (a) Plot of auroral acceleration parameters for the outer magnetosphere hot electron source plasma, shown versus azimuth angle $\varphi_{i}$. The upper panel of the figure shows the minimum field-aligned voltage required to produce the upward field-aligned current, $\Phi_{\|}$, given by Eq. (10). As in Fig. 8, the central solid line corresponds to an assumed current layer width of $500 \mathrm{~km}$, while the upper and lower dashed lines represent boundary widths of 250 and $1000 \mathrm{~km}$, respectively. The middle panel similarly shows the minimum radial distance of the top of the accelerating voltage drop, in units of ionospheric radial distance (essentially the planetary radius), given by Eq. (11). The lower panel then shows the energy flux $E_{f}$ associated with the precipitating accelerated electrons, given by Eq. (12). The horizontal bars at the top of the panels indicate the merging gap (MG) and adiaroic regions around the polar cap boundary.

and Richardson and Sittler (1990), using Voyager plasma data. These data show that the hot plasma electrons that extend along the field lines to low altitudes have a typical number density of $\sim 0.2 \mathrm{~cm}^{-3}$ and a thermal energy of $\sim 150 \mathrm{eV}$ in this region, as previously employed by Cowley and Bunce (2003) and Cowley et al. (2004b). Substituting into Eq. (9), these parameters yield a limiting current density of $\sim 66 \mathrm{nA} \mathrm{m}^{-2}$ which is thus much greater than the maximum $\sim 13 \mathrm{nA} \mathrm{m}^{-2}$ upward-directed field-aligned current in the middle magnetosphere shown in Fig. 6. These currents do not therefore require field-aligned acceleration of magnetospheric electrons, in conformity with the previous conclusions of Cowley and Bunce (2003) and Cowley et al. (2004b). We can also compute the energy flux associated with these precipitating electrons, given by Eq. (13), which is $\sim 0.020 \mathrm{Wm}^{-2}$. A simple "rule of thumb" is that $1 \mathrm{~mW} \mathrm{~m}^{-2}$ of electron energy input into the upper atmosphere results in $\sim 10 \mathrm{kR}$ of auroral UV output (Waite et al., 1983; Rego et al., 1994). Thus electron precipitation with this energy flux would result in an UV emission of only $\sim 0.2 \mathrm{kR}$, below current $\sim 1 \mathrm{kR}$ thresholds of detectability. We do not exclude the possibility of weak structured auroras occurring in this region, however, associated with smaller-scale flow features mentioned above and investigated by Cowley and Bunce (2003), but which are not included here.

We thus now consider the auroras associated with the upward-directed currents on the polar cap boundary. We note, however, that Knight's (1973) auroral acceleration theory outlined in Sect. 3.3 relates to the field-aligned current density, while our model yields only the field-aligned sheet current intensity at the boundary. In this case we simply make an assumption about the width of the current layer based on observations, and then check for model consistency with observed parameters such as the total auroral power. As discussed previously by Cowley et al. (2004a), the aurorae observed around (what is taken to be) the region of open field lines, have typical latitudinal widths of $\sim 300-1000 \mathrm{~km}$. Here we therefore present results for a range of boundary widths, from 250 to $1000 \mathrm{~km}$. In Fig. 8 we thus show the current density $j_{\| i}$ around the polar cap boundary for this 


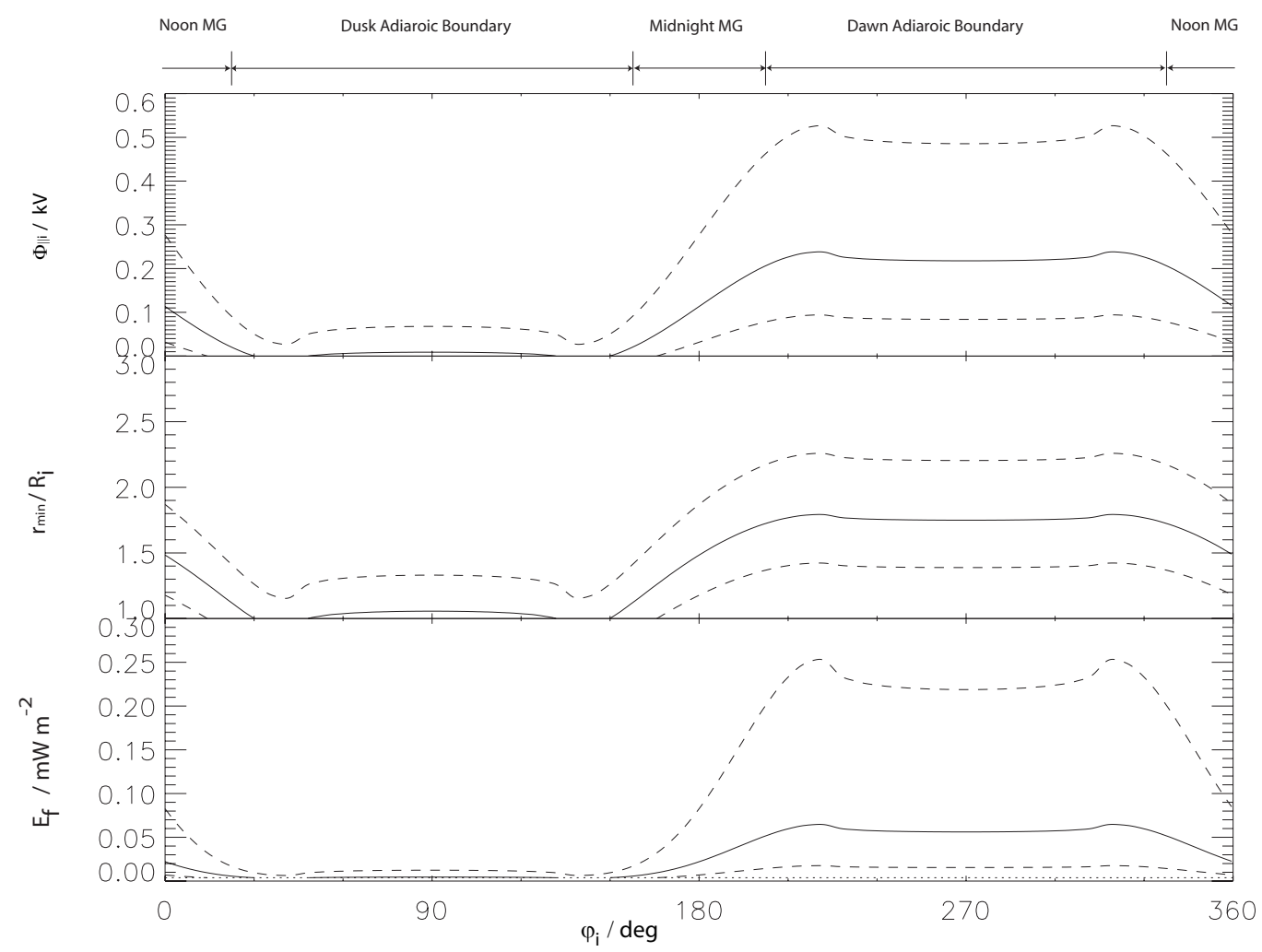

Fig. 9b. Figure continued. (b) As for Fig. 9a, but for the magnetosheath source plasma. The horizontal dotted line in the bottom panel shows the unaccelerated electron energy flux given by Eq. (13). This was too small to show in Fig 9a.

range of widths. The solid line shows the current density for the centre width of $500 \mathrm{~km}$, while for the upper and lower dashed lines the widths are a factor of two smaller $(250 \mathrm{~km})$ and larger $(1000 \mathrm{~km})$ respectively. The same line format will also be adopted for the auroral parameters shown below in Fig. 9. The current densities at dawn and dusk for each of these widths are given in Table 1 for easy reference, spanning $\sim 100-400 \mathrm{nA} \mathrm{m}^{-2}$ at dawn, and $\sim 20-80 \mathrm{nA} \mathrm{m}^{-2}$ at dusk.

These boundary currents may be carried partially in some lower latitude portion by hot electrons from the outer magnetosphere, with a number density of $\sim 0.01 \mathrm{~cm}^{-3}$ and a thermal energy of $\sim 1 \mathrm{keV}$ according to Voyager measurements (Richardson, 1986, 1995), and partially in some higher-latitude portion by magnetosheath (cusp) electrons with a number density of $\sim 0.2 \mathrm{~cm}^{-3}$ and a thermal energy of $\sim 50 \mathrm{eV}$ (Sittler et al., 1983). The outer magnetospheric source has a limiting current from Eq. (9) of $\sim 8.5 \mathrm{nA} \mathrm{m}^{-2}$, shown in Fig. 8 by the horizontal dot-dashed line. The limiting energy flux from Eq. (13) is $\sim 0.017 \mathrm{~mW} \mathrm{~m}^{-2}$, which we again note would by itself produce only a belowthreshold auroral UV intensity of $\sim 0.17 \mathrm{kR}$. The magnetosheath source correspondingly has a limiting current density of $\sim 37.8 \mathrm{nA} \mathrm{m}^{-2}$, shown in Fig. 8 by the horizontal dotted line, and an even lower energy flux of $\sim 0.0038 \mathrm{~mW} \mathrm{~m}^{-2}$. Comparison of these limiting current densities with the model values in Fig. 8 shows that auroral acceleration of both sources is required for all current layer widths at dawn. At some points along the dusk adiaroic boundary however, the current density falls below the threshold for acceleration of magnetosheath electrons for current layer widths of 500 and $1000 \mathrm{~km}$.

We now discuss the implications of these results for fieldaligned electron acceleration and aurorae. Figure 9a shows the three auroral parameters introduced in Sect. 3.3, computed using the above outer magnetosphere hot electron source, plotted versus azimuth in a similar format to Fig. 8 . The values at dawn and dusk are also given in Table 1 for each of the boundary widths. The top panel shows the minimum field-aligned voltage $\Phi_{\|}$required to produce the upward current, given by Eq. (10). The values at dawn are $\sim 10-50 \mathrm{kV}$, a factor of $\sim 5$ larger than those at dusk, which are $2-10 \mathrm{kV}$. The middle panel shows the minimum radial distance of the top of the accelerating voltage drop, in units of ionospheric radial distance, given by Eq. (11). The values are $\sim 2-4 R_{S}$ at dawn, and $\sim 1.5-2 R_{S}$ at dusk. The bottom panel shows the energy flux associated with the precipitating electrons, given by Eq. (12). The dawn-side energy fluxes are $\sim 2-20 \mathrm{~mW} \mathrm{~m}^{-2}$, sufficient to produce discrete auroral intensities of $\sim 20-200 \mathrm{kR}$ respectively. On the dusk side, however, the energy fluxes are strongly reduced to 
Table 1. Current density and auroral parameters at dawn and dusk on the polar cap boundary, for three assumed values of the width of the current layer.

\begin{tabular}{|c|c|c|c|c|c|c|}
\hline \multirow{2}{*}{$\begin{array}{l}\text { Current layer width } \\
\text { Local time }\end{array}$} & \multicolumn{2}{|c|}{$250 \mathrm{~km}$} & \multicolumn{2}{|c|}{$500 \mathrm{~km}$} & \multicolumn{2}{|c|}{$1000 \mathrm{~km}$} \\
\hline & Dawn & Dusk & Dawn & Dusk & Dawn & Dusk \\
\hline \multicolumn{7}{|l|}{ Current density } \\
\hline$j_{\| i} / \mathrm{nAm}^{-2}$ & 400 & 80 & 200 & 40 & 100 & 20 \\
\hline \multicolumn{7}{|c|}{ Auroral parameters for outer magnetosphere source } \\
\hline$\Phi_{\|} / \mathrm{kV}$ & 48 & 10 & 23 & 5.0 & 11 & 2.0 \\
\hline$r_{\min } / R_{i}$ & 3.7 & 2.2 & 2.9 & 1.7 & 2.2 & 1.4 \\
\hline$E_{f} / \mathrm{mW} \mathrm{m}^{-2}$ & 22 & 1.5 & 5.0 & 0.5 & 1.5 & 0.2 \\
\hline \multicolumn{7}{|c|}{ Auroral parameters for magnetosheath source } \\
\hline$\Phi_{\|} / \mathrm{kV}$ & 0.5 & 0.05 & 0.22 & 0.01 & 0.08 & 0.0 \\
\hline$r_{\min } / R_{i}$ & 2.2 & 1.4 & 1.7 & 1.1 & 1.4 & 1.0 \\
\hline$E_{f} / \mathrm{mW} \mathrm{m}^{-2}$ & 0.25 & 0.01 & 0.06 & 0.005 & 0.02 & 0.0 \\
\hline
\end{tabular}

$\sim 0.2-2 \mathrm{~mW} \mathrm{~m}^{-2}$, yielding auroral intensities of $\sim 2-20 \mathrm{kR}$, respectively. These strong variations of the electron energy flux with the width of the current layer, and between dawn and dusk, result from the fact that the energy flux is proportional to the square of the current density in Eq. (12).

Figure $9 \mathrm{~b}$ shows the same parameters for the magnetosheath electron source, with values at dawn and dusk again tabulated in Table 1. In this case, the values of the accelerating voltage, minimum acceleration region height, and precipitating energy flux are all significantly smaller than for the outer magnetosphere source due to the increased density and decreased temperature of the unaccelerated source electrons. The minimum field-aligned voltages shown in the upper panel peak at $\sim 0.1-0.5 \mathrm{kV}$ at dawn, and are about a factor of ten smaller at dusk. The minimum radial distance of the accelerating voltage drop in the middle panel also shows reduced values, generally below $\sim 2 R_{S}$. Similarly, the energy fluxes shown in the lower panel lie in the range $\sim 0.02-$ $0.2 \mathrm{~mW} \mathrm{~m}^{-2}$ at dawn, yielding auroral emissions in the range $\sim 0.2-2 \mathrm{kR}$, while on the dusk side the values are an order of magnitude lower, such that even the largest flux would yield an aurora too faint to be detected above present background levels of $\sim 1 \mathrm{kR}$. The horizontal dotted line in the bottom panel shows the unaccelerated electron energy flux for the magnetosheath source, $\sim 0.0038 \mathrm{~mW} \mathrm{~m}^{-2}$ as given by Eq. (13), that would yield an aurora of only $\sim 0.04 \mathrm{kR}$.
Combining together the results shown in Figs. 9a and $b$, and tabulated in Table 1, it can be seen that the currents at the polar cap boundary will be associated with bright aurorae in some equatorward portion where the current is carried by outer magnetosphere electrons, typically $\sim 50 \mathrm{kR}$ at dawn and $\sim 5 \mathrm{kR}$ at dusk, with weaker aurorae in some poleward portion where the current is carried by magnetosheath electrons, typically $\sim 1 \mathrm{kR}$ at dawn and decreasing below threshold at dusk. However, since such structure is at the limit of what can presently be spatially resolved, the observed boundary aurorae should clearly be dominated by that produced by the accelerated outer magnetosphere electrons, with the above parameters.

Finally, integration of the energy flux values across the current layer and around the open-closed field-line boundary yields the total power of the electrons precipitating in the boundary region. The total precipitating powers for the three current layer widths and both sources are given in Table 2. With $15 \%$ energy efficiency, these translate to UV output powers which are also given. For definiteness, the values given in the table assume that the whole of the current is carried by the given source in each case. It can be seen that for the centre width of $500 \mathrm{~km}$ the precipitating electron power for the outer magnetosphere source is $\sim 100 \mathrm{GW}$, while that for the magnetosheath source is only $\sim 1 \mathrm{GW}$. The corresponding UV output powers are $\sim 15$ and $\sim 0.2 \mathrm{GW}$, 
Table 2. Total precipitating electron power around the polar cap boundary, together with the auroral UV output, for various assumed current layer widths, and two magnetospheric electron source populations. For definiteness, the values given in the table assume that the whole of the current is carried by the given source in each case.

\begin{tabular}{|c|c|c|c|}
\hline Current layer width & $250 \mathrm{~km}$ & $500 \mathrm{~km}$ & $1000 \mathrm{~km}$ \\
\hline \multicolumn{4}{|l|}{ Outer magnetosphere source } \\
\hline $\begin{array}{l}\text { Precipitating electron power } \\
(\mathrm{GW})\end{array}$ & 204 & 102 & 51.6 \\
\hline UV output power (GW) & 30.6 & 15.3 & 7.75 \\
\hline \multicolumn{4}{|l|}{ Magnetosheath source } \\
\hline $\begin{array}{l}\text { Precipitating electron power } \\
(\mathrm{GW})\end{array}$ & 2.32 & 1.22 & 0.72 \\
\hline UV output power (GW) & 0.35 & 0.18 & 0.11 \\
\hline
\end{tabular}

respectively. For other widths the powers vary approximately inversely with the width. Clearly, the total powers for the outer magnetosphere source are far greater than those for the magnetosheath, such that the overall power will generally be dominated by the former contribution. For example, if we assume for simplicity that half the current is carried by electrons from the outer magnetospheric source and half by electrons from the magnetosheath, we find total precipitating electron powers of $\sim 103, \sim 51.9$, and $\sim 26.4 \mathrm{GW}$ for the 250 , 500 and $1000 \mathrm{~km}$ boundary widths respectively, yielding UV output powers of $\sim 15.5, \sim 7.79$, and $\sim 3.96 \mathrm{GW}$.

We now briefly consider the relationship of these results to the observed properties of the aurorae at Saturn. In overall terms, observations show that the auroral brightness typically lies in the range $\sim 1-100 \mathrm{kR}$, while the total auroral output power generally varies from a few $\mathrm{GW}$ to a few tens of GW (Gérard et al., 1995, 2004; Trauger et al., 1998; Cowley et al., 2004a; Clarke et al., 2005; Grodent et al., 2005; Badman et al., 2005). The values derived from our model lie in similar ranges, and are thus in basic accord with the observations. Dawn enhancements of the aurorae compared with dusk are also commonly observed, though not invariably so. Of course, our model has been designed to illustrate particular conditions in which the Dungey cycle is active, specifically compression region conditions in the solar wind when Dungey-cycle driving can reach averaged values of a few hundred $\mathrm{kV}$ over a few days, as shown by the results presented by Jackman et al. (2004) and Badman et al. (2005). Examination of auroral images obtained under such conditions during the joint Cassini-HST campaign in January 2004 (e.g. "visit 13"), do indeed show a substantial dawn-dusk asymmetry as indicated by our model, with auroral brightnesses of $\sim 10-50 \mathrm{kR}$, and total auroral output powers of $\sim 15 \mathrm{GW}$ (Clarke et al., 2005; Grodent et al., 2005; Badman et al., 2005). Our model thus also appears to be in basic agreement with these more specific observations. However, the model is certainly not expected to describe all circumstances, particularly solar wind rarefaction region conditions when the IMF is very weak, such that Dungey-cycle flow may become negligible for extended intervals.

We finally briefly address the issue of why it is that Dungey-cycle driving at Saturn produces a strong dawn-dusk auroral asymmetry, while no such zeroth order effect is observed at Earth where it is known that the Dungey cycle dominates (e.g. Cowley et al., 2003). Basically, the Dungey cycle twin-vortex flow is associated with field-aligned currents flowing on the open field boundary and on the equatorward boundary of the twin vortex, which are opposite at dawn and dusk, and opposite on the two boundaries. At Earth, these are termed the "region-1" and "region-2" field-aligned currents, respectively, and are directed upward at dusk and downward at dawn on the open field boundary, and vice-versa on the equatorward boundary. Discrete aurorae associated with upward field-aligned currents at Earth may thus be present both at dusk ("region-1") and dawn ("region-2"), joined by the upward currents of the Harang discontinuity across midnight, and augmented also by diffuse auroral precipitation from trapped hot plasma on closed flux tubes. At Saturn, these same currents are present, as in our model (though their directions are reversed compared with Earth due to the differing polarity of the planetary magnetic field), but they are now superposed on unidirectional currents flowing at the same boundaries associated with the shears in the rotational flow. These latter currents are directed upward on the open field boundary, and downward on the equatorward boundary, and as we have indicated here, they are sufficiently strong that 
usually the Dungey-cycle currents may modulate their magnitude but not reverse their sense. In this case discrete auroras associated with upward currents are confined to the open field boundary, where they are modulated in local time by the Dungey-cycle flow as in our model, while upward currents and discrete auroras are not expected to occur on the equatorward boundary. Of course, the overall auroral display at Saturn may also include some more uniform contribution from diffuse precipitation of trapped hot plasma on closed flux tubes, in the region just equatorward of the open field boundary.

\section{Summary}

In this paper we have proposed a simple model of the plasma flow and current in Saturn's polar ionosphere. This builds on the previous quantitative axi-symmetric model of Cowley et al. (2004b), by including the dawn-dusk asymmetries present when both the solar wind and internally driven processes are active, as discussed qualitatively by Cowley et al. (2004a). The model parameters have been guided by Voyager plasma velocity measurements on closed field lines (e.g. Richardson, 1986, 1995; Richardson and Sittler, 1990), by groundbased IR Doppler measurements of ionospheric flows in the polar cap (Stallard et al., 2004), and by remote sensing studies of the planet's aurorae using the HST (Gérard et al., 1995, 2004; Cowley et al., 2004a; Prangé et al., 2004).

The physical nature of the flow pattern in the model consists of elements which are intended to represent subcorotational plasma flow in the middle magnetosphere resulting from plasma pick-up and radial transport from internal sources, and the Vasyliunas- and Dungey-cycles of convection at higher latitudes. For theoretical convenience, the overall flow pattern representing these behaviours is expressed as the sum of two components. The first is a sub-corotating purely rotational flow varying with co-latitude, which is essentially similar to the axi-symmetric model of Cowley et al. (2004b). Dawn-dusk flow asymmetry is then introduced by vectorially adding a second flow component, consisting of a sun-aligned twin vortex, which provides anti-sunward flow across the polar cap, and return sunward flow via dawn and dusk confined to the outer magnetosphere ring. The antisunward flow across the polar cap enhances the rotational flow at dusk and diminishes it at dawn, while the return sunward flow in the outer magnetosphere enhances the rotational flow at dawn and diminishes it at dusk. The action of the twin-vortex flow is thus to increase the flow shears at both the polar cap and outer magnetosphere boundaries at dawn, while reducing them at both boundaries at dusk. The voltage associated with the twin vortex is just the Dungey cycle voltage associated with anti-sunward transport across the polar cap. Based on an analysis of upstream interplanetary Cassini data, Jackman et al. (2004) estimated this voltage to have typical values of $\sim 50 \mathrm{kV}$, with peaks of up to a few hundred $\mathrm{kV}$.
Here we have modelled active Dungey cycle conditions such as occur during solar wind compression regions, and have thus chosen a value of $200 \mathrm{kV}$. The voltage in the Vasyliunas cycle has been chosen to be comparable to the Dungey cycle, equal to $275 \mathrm{kV}$.

We then consider the height-integrated Pedersen current driven in the polar ionosphere by the plasma flow in the neutral atmosphere rest frame, and the field aligned currents that relate to its divergence. In our calculation we assumed a constant value of the effective height-integrated Pedersen conductivity of 1 mho on the basis of the results of Bunce et al. (2003), such that the currents calculated may be simply linearly scaled to other choices. For uniform conductivity, the Hall current closes wholly in the ionosphere, such that the field-aligned currents are determined only by the divergence of the Pedersen current. The rotational flow component then yields distributed field aligned currents flowing through the region of interest, which are essentially similar to those modelled by Cowley et al. (2004b), except that here the currents flowing on the polar cap and outer magnetosphere boundaries are simplified to sheet currents, rather than being resolved into structures of finite width. The distributed field-aligned currents in the polar cap and outer magnetosphere are downward-directed, and of magnitude $\sim 15$ and $\sim 5 \mathrm{nA} \mathrm{m}^{-2}$ respectively. The field-aligned current then reverses sense in the middle magnetosphere, with a peak upward-directed current of $\sim 13 \mathrm{nA} \mathrm{m}^{-2}$ at $\sim 21.5^{\circ}$ co-latitude. The sheet currents associated with the rotational flow are then upward-directed on the polar cap boundary, and downward-directed on the outer magnetosphere boundary. By its construction, however, the twin-vortex flow produces no distributed field-aligned currents, but only sheet currents on the boundaries. On the polar cap boundary these are directed upwards at dawn and downwards at dusk, and vice versa at the outer magnetosphere boundary, thus modulating the axi-symmetric sheet currents of the rotational flow component. The combined upward currents on the polar cap boundary peak at $\sim 0.10 \mathrm{~A} \mathrm{~m}^{-1}$ at dawn, and have a minimum of $\sim 0.02 \mathrm{~A} \mathrm{~m}^{-1}$ at dusk, while the downward currents on the outer magnetosphere boundary peak at $\sim 0.05 \mathrm{~A} \mathrm{~m}^{-1}$ at dawn and fall to zero at dusk.

We finally consider the implications of the upwarddirected field aligned currents in the model for electron acceleration and aurorae. Downward-directed field-aligned acceleration of magnetospheric electrons is required if the upward-directed field-aligned current density exceeds the maximum value that can be provided by the unaccelerated electron population. The latter maximum current depends on the density and temperature of the source plasma, which is taken to be the hot electron component in the middle magnetosphere, while at the polar cap boundary we consider two sources, the hot outer magnetosphere electrons and cooler denser electrons of the magnetosheath. We firstly find that the broadly-distributed upward-directed field aligned currents associated with sub-corotation in the middle 
magnetosphere are too small to require field-aligned acceleration of magnetospheric electrons, in conformity with the prior conclusions of Cowley and Bunce (2003) and Cowley et al. (2004b), and that the energy fluxes of the unaccelerated electrons are too weak to produce significant aurorae. This does not preclude the existence of weak structured auroras in this region, however, associated with small-scale flow features not included in our model (Cowley and Bunce, 2003). Interest then focuses on the upward-directed currents along the polar cap boundary. Our model only yields the sheet current intensity along this boundary, however, so we need to assume a width of the current layer in order to estimate the current density and resulting auroral accelerations. We chose to model a range of observationally-motivated current sheet widths from 250 to $1000 \mathrm{~km}$, yielding upward current densities of $\sim 100-400 \mathrm{nA} \mathrm{m}^{-2}$ at dawn and $\sim 20-80 \mathrm{nA} \mathrm{m}^{-2}$ at dusk. These values compare with maximum current densities for the unaccelerated population of $\sim 8.5 \mathrm{nA} \mathrm{m}^{-2}$ for the outer magnetosphere source, and $\sim 38 \mathrm{nA} \mathrm{m}^{-2}$ for the magnetosheath source. Auroral electron acceleration is thus required at all local times for all boundary widths considered for the outer magnetosphere source, more so at dawn than at dusk, while for the magnetosheath source the current density falls below the maximum that can be carried by the unaccelerated electrons for the larger widths at dusk.

For the outer magnetosphere source the accelerating voltages peak in the dawn sector at $\sim 10-50 \mathrm{kV}$ for the three boundary widths considered, yielding precipitating energy fluxes of $\sim 2-20 \mathrm{~mW} \mathrm{~m}^{-2}$. The associated auroral intensities are then $\sim 20-200 \mathrm{kR}$. On the dusk side the corresponding voltage and energy flux values fall to $\sim 2-10 \mathrm{kV}$ and $\sim 0.2-$ $2 \mathrm{~mW} \mathrm{~m}^{-2}$, yielding auroral intensities of $\sim 2-20 \mathrm{kR}$. The model thus suggests the presence of substantial dawn-dusk auroral asymmetry under the active Dungey-cycle conditions considered. For the magnetosheath source, the corresponding accelerating voltages and energy fluxes are much less, peaking in the dawn sector, for example, at $\sim 0.1-0.5 \mathrm{kV}$ and $\sim 0.02-0.2 \mathrm{~mW} \mathrm{~m}^{-2}$. The auroral energy deposited will thus usually be dominated by the portion of the boundary current carried by hot outer magnetosphere electrons. We have also integrated the energy flux values across the current layer and around the open-closed field line boundary, to yield the total UV output power. If we assume for simplicity that half the current is carried by each plasma source, we find total output powers of $\sim 5-15 \mathrm{GW}$.

Overall we find that the auroral brightnesses and total output powers derived here are in broad agreement with observations. Dawn-dusk asymmetry in the sense indicated is also found to be a common but not invariable feature of Saturn's auroras. Of course, the model discussed here has been developed to illustrate particular magnetospheric conditions, specifically intervals of steady strong Dungey-cycle driving, such as are likely to occur during solar wind compression regions when the IMF and magnetopause reconnection rates are strong. HST observations obtained under these conditions during the January 2004 campaign appear to be in good overall agreement with the theoretical results derived. However, the model will not apply in detail to other circumstances, such as solar wind rarefaction regions where the IMF is weak, and magnetospheric activity may be confined to episodic intervals of tail reconnection for example. Further development of the model to describe more diverse and time-dependent circumstances is thus warranted.

Acknowledgements. This work was supported by PPARC grant PPA/G/O/2003/00013. SWHC was supported during part of the study by PPARC Senior Fellowship PPA/N/S/2000/00197.

Topical Editor T. Pulkkinen thanks two referees for their help in evaluating this paper.

\section{References}

Badman, S. V., Bunce, E. J., Clarke, J. T., Cowley, S. W. H., Gérard, J.-C., Grodent, D., and Milan, S. E.: Open flux estimates in Saturn's magnetosphere during the January 2004 CassiniHST campaign, and implications for reconnection rates, J. Geophys. Res., 110, A11216, doi:10.1029/2005JA011240, 2005.

Boudouridis, A., Zesta, E., Lyons, L. R., Anderson, P. C., and Lummerzheim, D.: Magnetospheric reconnection driven by solar wind pressure fronts, Ann. Geophys., 22, 1367-1378, 2004.

Boudouridis, A., Zesta, E., Lyons, L. R., Anderson, P. C., and Lummerzheim, D.: Enhanced solar wind geoeffectiveness after a sudden increase in dynamic pressure during southward IMF orientation, J. Geophys. Res., 110, A05214, doi:10.1029/2004JA010704, 2005.

Bunce, E. J. and Cowley, S. W. H.: Divergence of the equatorial current in the dawn sector of Jupiter's magnetosphere: analysis of Pioneer and Voyager magnetic field data, Planet. Space Sci., 49, 1089-1113, 2001.

Bunce, E. J., Cowley, S. W. H., and Wild, J. A.: Azimuthal magnetic fields in Saturn's magnetosphere: Effects associated with plasma sub-corotation and the magnetopause-tail current system, Ann. Geophys., 21, 1709-1722, 2003.

Bunce, E. J., Cowley, S. W. H., Jackman, C. M., Clarke, J. T., Crary, F. J., and Dougherty, M. K.: Cassini observations of the interplanetary medium upstream of Saturn and their relation to Hubble Space Telescope auroral data, Adv. Space Res., in press, 2005a.

Bunce, E. J., Cowley, S. W. H., Wright, D. M., Coates, A. J., Dougherty, M. K., Krupp, N., Kurth, W. S., and Rymer, A. M.: In-situ observations of a solar wind compression-induced hot plasma injection in Saturn's tail, Geophys. Res. Lett., 32, L20S04, doi:10.1029/2005GL022888, 2005b.

Clarke, J. T., Gérard, J.-C., Grodent, D., Wannawichian, S., Gustin, J., Connerney, J., Crary, F., Dougherty, M., Kurth, W., Cowley, S. W. H., Bunce, E. J., Hill, T., and Kim, J.: Morphological differences between Saturn's ultraviolet aurorae and those of Earth and Jupiter, Nature, 433, 717-719, 2005.

Cowley, S. W. H. and Bunce, E. J.: Origin of the main auroral oval in Jupiter's coupled magnetosphere-ionosphere system, Planet. Space Sci., 49, 1067-1088, 2001.

Cowley, S. W. H. and Bunce, E. J.: Corotation-driven magnetosphere-ionosphere coupling currents in Saturn's magne- 
tosphere and their relation to the auroras, Ann. Geophys., 21, 1691-1707, 2003.

Cowley, S. W. H., Davies, J. A., Grocott, A., Khan, H., Lester, M., McWilliams, K. A., Milan, S. E., Provan, G., Sandholt, P. E., Wild, J. A., and Yeoman, T. K.: Solar wind-magnetosphereionosphere interactions in the Earth's plasma environment, Phil. Trans. A, 361, 113-126, 2003.

Cowley, S. W. H., Bunce, E. J., and Prangé, R.: Saturn's polar ionospheric flows and their relation to the main auroral oval, Ann. Geophys., 22, 1379-1394, 2004a.

Cowley, S. W. H., Bunce, E. J., and O'Rourke, J. M.: A simple quantitative model of plasma flows and currents in Saturn's polar ionosphere, J. Geophys. Res., 109 (A05212), doi:10.1029/2003JA010375, 2004b.

Cowley, S. W. H., Badman, S. V., Bunce, E. J., Clarke, J. T., Gérard, J.-C., Grodent, D., Jackman, C. M., Milan, S. E., and Yeoman, T. K.: Reconnection in a rotation-dominated magnetosphere and its relation to Saturn's auroral dynamics, J. Geophys. Res., 110, A02201, doi:10.1029/2004JA010796, 2005a.

Cowley, S. W. H., Alexeev, I. I., Belenkaya, E. S., Bunce, E. J., Cottis, C. E., Kalegaev, V. V., Nichols, J. D., Prangé, R., and Wilson, F. J.: A simple axi-symmetric model of magnetosphereionosphere coupling currents in Jupiter's polar ionosphere, J. Geophys. Res., 110, A11209, doi:10.1029/2005JA011237, $2005 b$.

Crary, F. J., Clarke, J. T., Dougherty, M. K., Hanlon, P. G., Hansen, K. C., Steinberg, J. T., Barraclough, B. L., Coates, A. J., Gérard, J.-C., Grodent, D., Kurth, W. S., Mitchell, D. G., Rymer, A. M., and Young, D. T.: Solar wind dynamic pressure and electric field as the main factors controlling Saturn's auroras, Nature, 433, 720-722, 2005.

Davis Jr., L. and Smith E. J.: A model of Saturn's magnetic field based on all available data, J. Geophys. Res., 95, 15 257-15 261, 1990.

Desch, M. D.: Evidence for solar wind control of Saturn radio emission, J. Geophys. Res., 87, 4549-4554, 1982.

Desch, M. D. and Rucker, H. O.: The relationship between Saturn kilometric radiation and the solar wind, J. Geophys. Res., 88, 8999-9006, 1983.

Dougherty, M. K., Achilleos, N., André, N., Arridge, C. S., Balogh, A., Bertucci, C., Burton, M. E., Cowley, S. W. H., Erdos, G., Giampieri, G., Glassmeier, K.-H., Khurana, K. K., Leisner, J., Neubauer, F. M., Russell, C. T., Smith, E. J., Southwood, D. J., and Tsurutani, B. T.: Cassini magnetometer observations during Saturn orbit insertion, Science, 307, 1266-1270, 2005.

Dungey, J. W.: Interplanetary field and the auroral zones, Phys. Rev. Lett., 6, 47-48, 1961.

Freeman, M. P. and Southwood, D. J.: The effect of magnetospheric erosion on mid-latitude and high-latitude ionospheric flows, Planet. Space. Sci., 36, 509-522, 1988.

Freeman, M. P., Ruohoniemi, J. M., and Greenwald, R. A.: The determination of time-stationary two-dimensional convection patterns with single-station radars, J. Geophys. Res., 96, 15735$15749,1991$.

Gérard, J.-C., Dols, V., Grodent, D., Waite, J. H., Gladstone, G. R., and Prangé, R.: Simultaneous observations of the saturnian aurora and polar haze with the HST/FOC, Geophys. Res. Lett., 22, 2685-2688, 1995.

Gérard, J.-C., Grodent, D., Gustin, J., Saglam, A., Clarke, J.
T., and Prangé, R.: Characteristics of Saturn's FUV aurora observed with the Space Telescope Imaging Spectrograph, J. Geophys. Res., J. Geophys. Res., 109, A09207, doi:10.1029/2004JA010513, 2004.

Grodent, D., Clarke, J. T., Kim, J., Waite Jr., J. H., and Cowley, S. W. H.: Jupiter's main oval observed with HST-STIS, J. Geophys. Res., 108 (A11), 1389, doi:10.1029/2003JA009921, 2003.

Grodent, D., Gérard, J.-C., Cowley, S. W. H., Bunce, E. J., and Clarke, J. T.: The global morphology of Saturn's southern ultraviolet aurora, J. Geophys. Res., 110, A07215, doi:10.1029/2004JA010983, 2005.

Hill, T. W.: Inertial limit on corotation, J. Geophys. Res., 84, 6554 6558, 1979.

Hill, T. W.: The jovian auroral oval, J. Geophys. Res., 106, 81018107, 2001.

Huang, T. S. and Hill, T. W.: Corotation lag of the jovian atmosphere, ionosphere and magnetosphere, J. Geophys. Res., 94, 3761-3765, 1989.

Isbell, J., Dessler, A. J., and Waite Jr., J. H.: Magnetospheric energization by interaction between planetary spin and the solar wind, J. Geophys. Res., 89, 10 716-10722, 1984.

Jackman, C. M., Achilleos, N., Bunce, E. J., Cowley, S. W. H., Dougherty, M. K., Jones, G. H., Milan, S. E., and Smith, E. J.: Interplanetary magnetic field at $\sim 9$ AU during the declining phase of the solar cycle and its implications for Saturn's magnetospheric dynamics, J. Geophys. Res., 109, A11203, doi:10.1029/2004JA010614, 2004.

Jackman, C. M., Achilleos, N., Bunce, E. J., Cecconi, B., Clarke, J. T., Cowley, S. W. H., Kurth, W. S., and Zarka, P.: Interplanetary conditions and magnetospheric dynamics during the Cassini orbit insertion fly-through of Saturn's magnetosphere, J. Geophys. Res., 110, A10212, doi:10.1029/2005JA011054, 2005.

Knight, S.: Parallel electric fields, Planet. Space Sci., 21, 741-750, 1973.

Krimigis, S. M., Mitchell, D. G., Hamilton, D. C., Krupp, N., Livi, S., Roelof, E. C., Dandouras, J., Armstrong, T. P., Mauk, B. H., Paranicas, C., Brandt, P. C., Bolton, S., Cheng, A. F., Choo, T., Gloeckler, G., Hayes, J., Hsieh, K. S., Ip, W.-H., Jaskulek, S., Keath, E. P., Kirsch, E., Kusterer, M., Lagg, A., Lanzerotti, L. J., LaVallee, D., Manweiler, J., McEntire, R. W., Rasmuss, W., Saur, J., Turner, F. S., Williams, D. J., and Woch, J.: Dynamics of Saturn's magnetosphere from MIMI during Cassini's orbital insertion, Science, 307, 1270-1273, 2005.

Kurth, W. S., Gurnett, D. A., Clarke, J. T., Zarka, P., Desch, M. D., Kaiser, M. L., Cecconi, B., Lecacheux, A., Farrell, W. M., Galopeau, P., Gérard, J.-C., Grodent, D., Prangé, R., Dougherty, M. K., and Crary, F. J.: An Earth-like correspondence between Saturn's auroral features and radio emission, Nature, 433, 722-725, 2005.

Lundin, R. and Sandahl, I.: Some characteristics of the parallel electric field acceleration of electrons over discrete auroral arcs as observed from two rocket flights, in Symposium on European Rocket Research, ESA SP-135, ESA, Noordwijk, The Netherlands, 125, 1978.

Milan, S. E., Cowley, S. W. H., Lester, M., Wright, D. M., Slavin, J. A., Fillingim, M., Carlson, C. W., and Singer, H. J.: Response of the magnetotail to changes in the open flux content of the magnetosphere, J. Geophys. Res., 109, A04220, doi:10.1029/2003JA010350, 2004. 
Milan, S. E., Bunce, E. J., Cowley, S. W. H., and Jackman, C. M.: Implications of rapid planetary rotation for the Dungey magnetotail of Saturn, J. Geophys. Res., 110, A02201, doi:10.1029/2004JA010716, 2005

Millward, G., Miller, S., Stallard, T., Achilleos, N., and Aylward, A. D.: On the dynamics of the jovian ionosphere and thermosphere. IV; Ion-neutral coupling, Icarus, 173, 200-211, 2005.

Ness, N. F., Acuña, M.H., Lepping, R. P., Connerney, J. E. P., Behannon, K. W., Burlaga, L. F., and Neubauer, F. M.: Magnetic field studies by Voyager 1: Preliminary results at Saturn, Science, 212, 211-217, 1981.

Pontius, D. H. and Hill, T. W.: Departure from corotation of the Io plasma torus - local plasma production, Geophys. Res. Lett., 12, 1321-1324, 1982.

Prangé, R., Pallier, L., Hansen, K. C., Howard, R., Vourladis, A., Courtin, R., and Parkinson, C.: A CME-driven interplanetary shock traced from the Sun to Saturn by planetary auroral storms, Nature, 432, 78-81, 2004.

Rego, D., Prangé, R., and Gérard, J. C.: Auroral Lyman $\alpha$ and $\mathrm{H}_{2}$ bands from the giant planets: 1 . Excitation by proton precipitation in the Jovian aurorae, J. Geophys. Res., 99, 17 075-17 094, 1994.

Richardson, J. D.: Thermal ions at Saturn: Plasma parameters and implications, J. Geophys. Res., 91, 1381-1389, 1986.

Richardson, J. D.: An extended plasma model for Saturn, Geophys. Res. Lett., 22, 1177-1180, 1995.

Richardson, J. D. and Sittler Jr., E. C.: A plasma density model for Saturn based on Voyager observations, J. Geophys. Res., 95, 12 019-12 031, 1990.

Richardson, J. D., Eviatar, A., McGrath, M. A., and Vasyliunas, V. M.: OH in Saturn's magnetosphere: Observations and theory, J. Geophys. Res., 103, 20 245-20 255, 1998.

Saur, J., Mauk, B. H., Kassner, A., and Neubauer, F. M.: A model for the azimuthal plasma velocity in Saturn's magnetosphere, J. Geophys. Res., 109, A05217, doi:10.1029/2003JA010207, 2004.
Sittler Jr., E. C., Ogilvie, K. W., and Scudder, J. D.: Survey of lowenergy plasma electrons in Saturn's magnetosphere: Voyagers 1 and 2, J. Geophys. Res., 88, 8847-8870, 1983.

Southwood, D. J. and Kivelson, M. G.: A new perspective concerning the influence of the solar wind on Jupiter, J. Geophys. Res. 106, 6123-6130, 2001.

Stallard, T. S., Miller, S., Trafton, L. M., Geballe, T. R., and Joseph, R. D.: Ion winds in Saturn's southern auroral/polar region, Icarus, 167, 204-211, 2004.

Trauger, J. T., Clarke, J. T., Ballester, G. E., Evans, R. W., Burrows, C. J., Crisp, D., Gallagher III, J. S.,, Griffiths, R. E., Hester, J. J., Hoessel, J. G., Holtzman, J. A., Krist, J. E., Mould, J. R., Sahai, R., Scowen, P. A., Stapelfeldt, K. R., and Watson, A. M.: Saturn's hydrogen aurora: Wide field and planetary camera 2 imaging from the Hubble Space Telescope, J. Geophys. Res., 103, $20237-20244,1998$.

Vasyliunas, V. M.: Plasma distribution and flow, in Physics of the Jovian Magnetosphere, edited by: Dessler, A. J., Cambridge Univ. Press, Cambridge, UK, 395, 1983.

Waite, J. H., Cravens, T. E., Kozyra, J., Nagy, A. F., Atreya, S. K., and Chen, R. H.: Electron precipitation and related aeronomy of the Jovian thermosphere and ionosphere, J. Geophys. Res., 88, 6143-6163, 1983.

Young, D. T., Berthelier, J.-J., Blanc, M., Burch, J. L., Bolton, S., Coates, A. J., Crary, F. J., Goldstein, R., Grande, M., Hill, T. W., Johnson, R. E., Baragiola, R. A., Kelha, V., McComas, D. J., Mursula, K., Sittler, E. C., Svenes, K. R., Szegö, K., Tanskanen, P., Thomsen, M. F., Bakshi, S., Baraclough, B. L., Bebesi, Z., Delapp, D., Dunlop, M. W., Gosling, J. T., Furman, J. D., Gilbert, L. K., Glenn, D., Holmlund, C., Illiano, J.M., Lewis, G. R., Linder, D. R., Maurice, S., McAndrews, H. J., Narheim, B. T., Pallier, E., Reisenfeld, D., Rymer, A. M., Smith, H. T., Tokar, R. L., Vilppola, J., and Zinsmeyer, C.: Composition and dynamics of plasma in Saturn's magnetosphere, Science, 307, 1262-1266, 2005. 